\title{
Resonant relaxation in electroweak baryogenesis
}

\author{
Christopher Lee, ${ }^{*}$ Vincenzo Cirigliano, ${ }^{\dagger}$ and Michael J. Ramsey-Musolf ${ }^{\ddagger}$ \\ California Institute of Technology, Pasadena, California 91125, USA
}

(Received 15 January 2005; published 22 April 2005)

\begin{abstract}
We compute the leading, chiral charge-changing relaxation term in the quantum transport equations that govern electroweak baryogenesis using the closed time path formulation of nonequilibrium quantum field theory. We show that the relaxation transport coefficients may be resonantly enhanced under appropriate conditions on electroweak model parameters and that such enhancements can mitigate the impact of similar enhancements in the $C P$-violating source terms. We also develop a power counting in the time and energy scales entering electroweak baryogenesis and include effects through second order in ratios $\epsilon$ of the small and large scales. We illustrate the implications of the resonantly enhanced $\mathcal{O}\left(\epsilon^{2}\right)$ terms using the Minimal Supersymmetric Standard Model, focusing on the interplay between the requirements of baryogenesis and constraints obtained from collider studies, precision electroweak data, and electric dipole moment searches.
\end{abstract}

DOI: 10.1103/PhysRevD.71.075010

PACS numbers: 12.60.Jv, 98.80.Cq

\section{INTRODUCTION}

The origin of the baryon asymmetry of the Universe (BAU) remains an important, unsolved problem for particle physics and cosmology. Assuming that the Universe was matter-antimatter symmetric at its birth, it is reasonable to suppose that interactions involving elementary particles generated the BAU during subsequent cosmological evolution. As noted by Sakharov [1], obtaining a nonzero BAU requires both a departure from thermal equilibrium as well as the breakdown of various discrete symmetries: baryon number $(B)$ conservation, charge conjugation $(C)$ invariance, and invariance under the combined $C$ and parity $(P)$ transformations. ${ }^{1}$ The standard model (SM) of strong and electroweak interactions satisfies these conditions and could, in principle, explain the observed size of the BAU:

$$
Y_{B} \equiv \frac{\rho_{B}}{s}= \begin{cases}(7.3 \pm 2.5) \times 10^{-11}, & \text { BBN } \\ (9.2 \pm 1.1) \times 10^{-11}, & \text { WMAP }\end{cases}
$$

where $\rho_{B}$ is the baryon number density, $s$ is the entropy density of the Universe, and where the values shown correspond to $95 \%$ confidence level results obtained from Big Bang Nucleosynthesis (BBN) [2] and the Wilkinson Microwave Anisotropy Probe (WMAP) [3], respectively. In practice, however, neither the strength of the first-order electroweak phase transition in the SM nor the magnitude of SM $C P$-violating interactions are sufficient to prevent washout of any net baryon number created by $B$-violating electroweak sphaleron transitions during the phase transition.

\footnotetext{
*Electronic address: leec@ @ theory.caltech.edu

†Electronic address: vincenzo@ caltech.edu

‡Electronic address: mjrm@caltech.edu

${ }^{1}$ Allowing for a breakdown of $C P T$ invariance relaxes the requirement of departure from thermal equilibrium.
}

The search for physics beyond the SM is motivated, in part, by the desire to find new particles whose interactions could overcome the failure of the SM to explain the BAU. From a phenomenological standpoint, a particularly attractive possibility is that masses of such particles are not too different from weak scale and that their interactions both strengthen the first-order electroweak phase transition and provide the requisite level of $C P$-violation needed for the BAU. Precision electroweak measurements as well as direct searches for new particles at the Tevatron and Large Hadron Collider may test this possibility, and experiment already provides rather stringent constraints on some of the most widely considered extensions of the SM. In the Minimal Supersymmetric Standard Model (MSSM), for example, present lower bounds on the mass of the lightest Higgs boson leave open only a small window for a sufficiently strong first-order phase transition, although this constraint may be relaxed by introducing new gauge degrees of freedom (see, e.g., [4,5]). Similarly, limits on the permanent electric dipole moments (EDMs) of elementary particles and atoms imply that the $C P$-violating phases in the MSSM must be unnaturally small $\left(\sim 10^{-2}\right)$. Whether such small phases (supersymmetric or otherwise) can provide for successful electroweak baryogenesis (EWB) has been an important consideration in past studies of this problem.

In order to confront phenomenological constraints on the parameters of various electroweak models with the requirements of EWB, one must describe the microscopic dynamics of the electroweak phase transition in a realistic way. Theoretically, the basic mechanism driving baryogenesis during the phase transition is well-established. Weak sphaleron transitions that conserve $B-L$ but change $B$ and $L$ individually are unsuppressed in regions of spacetime where electroweak symmetry is unbroken, while they become exponentially suppressed in regions of broken symmetry. Net baryon number is captured by expanding regions of broken symmetry ("bubbles"). Given 
sufficiently strong $C$ and $C P$-violation as well as departure from thermal equilibrium, the nonzero $B$ generated outside the bubble cannot be entirely washed out by elementary particle interactions that occur at the phase boundary. The baryon number density, $\rho_{B}$, is governed by a diffusion equation of the form:

$$
\partial_{t} \rho_{B}(x)-D \nabla^{2} \rho_{B}(x)=-\Gamma_{\mathrm{ws}} F_{\mathrm{ws}}(x)\left[n_{L}(x)+R \rho_{B}(x)\right],
$$

where $D$ is the diffusion coefficient for baryon number, $\Gamma_{\text {ws }}$ is the weak sphaleron transition rate, $F_{\mathrm{ws}}(x)$ is a sphaleron transition profile function that goes to zero inside the regions of broken electroweak symmetry and asymptotically to unity outside, $R$ is a relaxation coefficient for the decay of baryon number through weak sphaleron transitions, and $n_{L}(x)$ is the number density of left-handed doublet fields created by "fast" chirality-changing processes (see, e.g., [6]). Thus, in order to obtain nonzero $\rho_{B}$ inside the bubble of broken electroweak symmetry, the left-handed density $n_{L}$ must be nonvanishing in the plasma at the phase boundary and possibly beyond into the region of unbroken symmetry.

In effect, $n_{L}(x)$ acts as a seed for the $B$-changing weak sphaleron transitions, and its spacetime profile is determined by the $C P$-violating sources and the quantum transport of various charges in the nonequilibrium environment of the plasma. Typical treatments of these dynamics involve writing down a set of coupled quantum transport equations (QTEs) for the relevant charges, estimating (or parametrizing) the relevant transport coefficients, and solving the system of equations under the appropriate boundary conditions.

Among the developments in the past decade or so which have made significant impacts on this program, we identify two that form the basis of our investigation in this work. First, the authors of Ref. [7] noted that diffusion of chiral charge ahead of the advancing phase transition boundary into the region of unbroken symmetry could enhance the impact of baryon number-changing sphaleron processes, thereby leading to more effective EWB. The second, perhaps less widely appreciated, development has been the observation by the author of Ref. [8] that the application of equilibrium quantum field theory (QFT) to transport properties in the plasma is not necessarily appropriate. In contrast to equilibrium quantum dynamics, the time evolution of quantum states during the phase transition is nonadiabatic. Consequently, scattering processes that drive quantum transport are no longer Markovian, but rather retain some memory of the system's quantum evolution. Using the closed time path (CTP) formulation of nonequilibrium QFT [9] to compute the $C P$-violating source terms in the plasma for the MSSM, the author of Ref. [8] found that these "memory effects" may lead to significant resonant enhancements (of order $10^{3}$ ) of the sources over their strength estimated in previous treatments (see, e.g.,
Ref. [10] and references therein). The authors of Ref. [11,12] subsequently found that performing an allorders summation of scattering from Higgs backgrounds reduces the size of the $C P$-violating sources to some extent, but that the resonant enhancements nonetheless persist. Taken at face value, these enhancements would imply that successful EWB could occur with significantly smaller $C P$-violating phases than previously believed, thereby evading the present and prospective limits obtained from EDMs.

To determine whether or not such conclusions are warranted, however, requires that one treat the other terms in the transport equations in the same manner as the $C P$-violating sources. Here, we attempt to do so, focusing on the terms that, in previous studies, have governed the relaxation of $n_{L}(x)$. In particular, chirality-changing Yukawa interactions with the Higgs fields and their spacetime varying vacuum expectation values (vevs) tend to wash out excess $n_{L}(x)$. In earlier studies - including those in which nonequilibrium QFT has been applied to the $C P$-violating sources - these relaxation terms were estimated using conventional quantum transport theory $[8,10-$ 12]. However, if the memory effects that enhance the $C P$-violating sources have a similar effect on these Yukawa terms, then the net effect on $\rho_{B}$ may not be as substantial as suggested in Refs. [8,11,12].

The goal of the present study is to address this question by developing a more comprehensive treatment of EWB using the CTP formulation of nonequilibrium QFT. In doing so, we follow the direction suggested in Ref. [8] and compute the transport coefficients of the chiral charges using the CTP formalism. To make the calculation more systematic, we identify the relevant energy and time scales that govern finite temperature, nonequilibrium dynamics and develop a power counting in the ratios of small to large scales (generically denoted here as $\epsilon$ ). As we show below, both the $C P$-violating sources and the driving relaxation terms first arise at $\mathcal{O}\left(\epsilon^{2}\right)$, and we truncate our analysis at this order. In contrast to the computation of the $C P$-violating sources, the derivation of the relaxation terms requires the use of finite density Green's functions. Given the resulting complexity, we consider here only the terms in the transport equations that previous authors have considered the dominant ones, and use our analysis of these terms to illustrate a method for obtaining a more comprehensive treatment of the QTEs. To make the phenomenological implications concrete, we focus on the MSSM, realizing, however, that one may need to include extensions of the MSSM in order to satisfy the requirements of a strong first-order phase transition. Finally, we also attempt to identify the different approximations that have entered previous treatments of EWB, such as the implicit truncation at a given order in $\epsilon$ and outline additional calculations needed to obtain a comprehensive treatment.

Based on our analysis, we find that under the same conditions that lead to resonant enhancements of the 
$C P$-violating sources, $S^{C P}$, one also obtains a similar, resonant enhancement of the driving chirality-changing transport coefficient, $\bar{\Gamma}$. Since $Y_{B} \sim S^{C P} / \sqrt{\bar{\Gamma}}$, resonant relaxation counteracts the enhanced sources, though some overall enhancement of EWB still persists. Consequently, it will be important in future work to study the other transport coefficients whose impact has been considered subleading, since they may be enhanced under conditions other than those relevant for the leading terms. From the standpoint of phenomenology, we also illustrate how the implications of EDM searches for EWB depends in a detailed way on the electroweak model of interest as well as results from collider experiments and precision electroweak data.

In presenting our study, we attempt to be somewhat pedagogical, since the methods are, perhaps, not generally familiar to either the practitioners of field theory or experimentalists. Most of the formal development appears in Sections II, III, and IV. In Section II we review the CTP formalism and its application to the QTEs and discuss in detail the formulation of density-dependent Green's functions. In Section III we compute the $C P$-violating source terms, providing a check of Ref. [8], as well as the transport coefficients of the chiral charge densities. Here, we also enumerate the approximations used to obtain a set of coupled, linear differential diffusion equations, discuss their limits of validity, and identify additional terms (usually assumed to be subleading) that we defer to a future study. In Section IV we solve these equations for the baryon density. A reader primarily interested in the phenomenological implications may want to turn directly to Section V, which gives illustrative numerical studies using the parameters of the MSSM. A discussion of the implications for EDMs also appears here. Section VI contains a summary and outlook, while several technical details appear in the Appendices.

\section{NONEQUILIBRIUM TRANSPORT: CTP FORMULATION}

In what follows, we treat all $C P$-violating and nontopological chirality-changing interactions perturbatively. ${ }^{2}$ In contrast to zero-temperature, equilibrium perturbation theory, however, the perturbative expansion under nonequilibrium, $T>0$ conditions requires the use of a more general set of Green's functions that take into account the nonadiabatic evolution of states as well as the presence of degeneracies in the thermal bath. Specifically, the matrix element of any operator $\mathcal{O}(x)$ in the interaction representation is given by:

$$
\left\langle n\left|S_{\text {int }}^{\dagger} T\left\{\mathcal{O}(x) S_{\text {int }}\right\}\right| n\right\rangle,
$$

\footnotetext{
${ }^{2}$ Sphaleron transitions, however, are manifestly nonperturbative, and we parametrize their effects in the standard way.
}

where

$$
S_{\text {int }}=T \exp \left(i \int d^{4} x \mathcal{L}_{\text {int }}\right)
$$

for an interaction Lagrangian $\mathcal{L}_{\text {int }}, T$ is the time-ordering operator, and $|n\rangle$ is an in-state. In ordinary, zerotemperature equilibrium field theory, the assumptions of adiabaticity and of nondegeneracy of the states $|n\rangle$ imply that the only impact of $S_{\text {int }}^{\dagger}$ is the introduction of an overall phase, allowing one to rewrite (3) as:

$$
\frac{\left\langle n\left|T\left\{\mathcal{O}(x) S_{\text {int }}\right\}\right| n\right\rangle}{\left\langle n\left|S_{\text {int }}\right| n\right\rangle} .
$$

This simplification is no longer valid for nonequilibrium $T>0$ evolution, and one must take into account the action of $S_{\text {int }}^{\dagger}$ appearing to the left of $\mathcal{O}(x)$ in (3). Doing so is facilitated by giving every field in $S_{\text {int }}$ and $S_{\text {int }}^{\dagger}$ a " + " and "-" subscript, respectively. The matrix element in (3) then becomes:

$$
\left\langle n\left|\mathcal{P}\left\{\mathcal{O}(x) \exp \left(i \int d^{4} x \mathcal{L}_{+}-i \int d^{4} x \mathcal{L}_{-}\right)\right\}\right| n\right\rangle,
$$

where the path ordering operator $\mathcal{P}$ indicates that all "+" fields appear to the right of all " - " fields, with the former being ordered according to the usual time-ordering prescription and the latter being anti-time-ordered [here, $\mathcal{O}(x)$ has been taken to be a "+" field]. Note that the two integrals in the exponential in (6) can be written as a single integral along a closed time path running from $-\infty$ to $+\infty$ and then back to $-\infty$.

Perturbation theory now proceeds from the matrix element (6) along the same lines as in ordinary field theory via the application of Wick's theorem, but with the more general $\mathcal{P}$ operator replacing the $T$ operator. As a result, one now has a set of four two-point functions, corresponding to the different combinations of "+" and "- " fields that arise from contractions. It is convenient to write them as a matrix $\tilde{G}(x, y)$ :

$$
\tilde{G}(x, y)=\left(\begin{array}{cc}
G^{t}(x, y) & -G^{<}(x, y) \\
G^{>}(x, y) & -G^{t}(x, y)
\end{array}\right),
$$

where

$$
\begin{aligned}
G^{>}(x, y)= & \left\langle\phi_{-}(x) \phi_{+}^{\dagger}(y)\right\rangle \\
G^{<}(x, y)= & \left\langle\phi_{-}^{\dagger}(y) \phi_{+}(x)\right\rangle \\
G^{t}(x, y)= & \left\langle T\left\{\phi_{+}(x) \phi_{+}^{\dagger}(y)\right\}\right\rangle=\theta\left(x_{0}-y_{0}\right) G^{>}(x, y) \\
& +\theta\left(y_{0}-x_{0}\right) G^{<}(x, y) \\
G^{\bar{t}}(x, y)= & \left\langle\bar{T}\left\{\phi_{-}(x) \phi_{-}^{\dagger}(y)\right\}\right\rangle=\theta\left(x_{0}-y_{0}\right) G^{<}(x, y) \\
& +\theta\left(y_{0}-x_{0}\right) G^{>}(x, y),
\end{aligned}
$$

and where the \langle\rangle denote ensemble averages,

$$
\langle\mathcal{O}(x)\rangle \equiv \frac{1}{Z} \operatorname{Tr}[\hat{\rho} \mathcal{O}(x)] .
$$


$\hat{\rho}$ is the density matrix containing information about the state of the system, and $Z=\operatorname{Tr}(\hat{\rho})$. In thermal equilibrium $\hat{\rho}$ is time-independent and is given by $\hat{\rho}=e^{-\beta\left(\mathrm{H}-\mu_{i} \mathrm{~N}_{i}\right)}$ for a grand-canonical ensemble. Note that the matrix $\tilde{G}(x, y)$ may be written more compactly as:

$$
\tilde{G}(x, y)_{a b}=\left\langle\mathcal{P}\left\{\phi_{a}(x) \phi_{b}^{\dagger}(y)\right\}\right\rangle\left(\tau_{3}\right)_{b b} .
$$

The presence of the $\tau_{3}$ factor is a bookkeeping device to keep track of the relative minus sign between the $\mathcal{L}_{+}$and $\mathcal{L}_{-}$terms in Eq. (6).

The path-ordered two-point functions satisfy the Schwinger-Dyson equations:

$$
\begin{aligned}
& \tilde{G}(x, y)=\tilde{G}^{0}(x, y)+\int d^{4} w \int d^{4} z \tilde{G}^{0}(x, w) \tilde{\Sigma}(w, z) \tilde{G}(z, y) \\
& \tilde{G}(x, y)=\tilde{G}^{0}(x, y)+\int d^{4} w \int d^{4} z \tilde{G}(x, w) \tilde{\Sigma}(w, z) \tilde{G}^{0}(z, y),
\end{aligned}
$$

where the " 0 " superscript indicates a noninteracting Green's function and where $\tilde{\Sigma}(x, y)$ is the matrix of interacting self-energies defined analogously to the $\tilde{G}(x, y)$. An analogous set of expressions apply for fermion Green's functions, with an appropriate insertion of -1 to account for anticommutation relations.

\section{A. Quantum Transport Equations from CTP Formalism}

The Schwinger-Dyson Eqs. (11) are the starting point for obtaining the transport equations governing $n_{L}(x)$. To do so, we follow Ref. [8] and apply the Klein-Gordon operator to $\tilde{G}(x, y)$. Using

$\left(\square_{x}+m^{2}\right) \tilde{G}^{0}(x, y)=\left(\square_{y}+m^{2}\right) \tilde{G}^{0}(x, y)=-i \delta^{(4)}(x-y)$

gives

$\left(\square_{x}+m^{2}\right) \tilde{G}(x, y)=-i \delta^{(4)}(x-y)-i \int d^{4} z \tilde{\Sigma}(x, z) \tilde{G}(z, y)$

$\left(\square_{y}+m^{2}\right) \tilde{G}(x, y)=-i \delta^{(4)}(x-y)-i \int d^{4} z \tilde{G}(x, z) \tilde{\Sigma}(z, y)$.

It is useful now to consider the $(a, b)=(1,2)$ components of these equations:

$$
\begin{aligned}
\left(\square_{x}+m^{2}\right) G^{<}(x, y)= & -i \int d^{4} z\left[\Sigma^{t}(x, z) G^{<}(z, y)\right. \\
& \left.-\Sigma^{<}(x, z) G^{\bar{t}}(z, y)\right] \\
\left(\square_{y}+m^{2}\right) G^{<}(x, y)= & -i \int d^{4} z\left[G^{t}(x, z) \Sigma^{<}(z, y)\right. \\
& \left.-G^{<}(x, z) \Sigma^{\bar{t}}(z, y)\right] .
\end{aligned}
$$

Subtracting Eq. (14b) from Eq. (14a) and multiplying through by $i$ gives

$$
\begin{aligned}
\left.i\left(\square_{x}-\square_{y}\right) G^{<}(x, y)\right|_{x=y \equiv X}= & i \partial_{\mu}^{X}\left[\left(\partial_{x}^{\mu}\right.\right. \\
& \left.\left.-\partial_{y}^{\mu}\right) G^{<}(x, y)\right]_{x=y \equiv X},
\end{aligned}
$$

However,

$$
\left.\left(\partial_{\mu}^{x}-\partial_{\mu}^{y}\right) G^{<}(x, y)\right|_{x=y \equiv X}=-i j_{\mu}(X),
$$

where $j_{\mu}(x)=i\left\langle: \phi^{\dagger}(x) \overleftrightarrow{\partial_{\mu}} \phi(x):\right\rangle \equiv(n(x), \vec{j}(x))$, since the "+" and "-" labels simply indicate the order in which the fields $\phi^{\dagger}(y)$ and $\phi(x)$ occur and may be dropped at this point. Finally, expressing $G^{t, \bar{t}}(x, y)$ and $\Sigma^{t, \bar{t}}(x, y)$ in terms of $\theta$-functions as in Eqs. (8), we obtain from Eq. (15):

$$
\begin{aligned}
\frac{\partial n}{\partial X_{0}}+\nabla \cdot \mathbf{j}(X)= & \int d^{3} z \int_{-\infty}^{X_{0}} d z_{0}\left[\Sigma^{>}(X, z) G^{<}(z, X)\right. \\
& -G^{>}(X, z) \Sigma^{<}(z, X) \\
& +G^{<}(X, z) \Sigma^{>}(z, X) \\
& \left.-\Sigma^{<}(X, z) G^{>}(z, X)\right] .
\end{aligned}
$$

Following similar steps, but taking the sum rather than the difference of the components of the Schwinger-Dyson equations involving the $S^{>}(x, y)$ component on the lefthand side (LHS), one obtains the analogous continuity equation for Dirac fermions:

$$
\begin{aligned}
\frac{\partial n}{\partial X_{0}}+\nabla \cdot \mathbf{j}(X)= & -\int d^{3} z \int_{-\infty}^{X_{0}} d z_{0} \operatorname{Tr}\left[\Sigma^{>}(X, z) S^{<}(z, X)\right. \\
& -S^{>}(X, z) \Sigma^{<}(z, X) \\
& +S^{<}(X, z) \Sigma^{>}(z, X) \\
& \left.-\Sigma^{<}(X, z) S^{>}(z, X)\right]
\end{aligned}
$$

where

$$
\begin{aligned}
& S_{\alpha \beta}^{>}(x, y)=\left\langle\psi_{-\alpha}(x) \bar{\psi}_{+\beta}(y)\right\rangle \\
& S_{\alpha \beta}^{<}(x, y)=-\left\langle\bar{\psi}_{-\beta}(y) \psi_{+\alpha}(x)\right\rangle,
\end{aligned}
$$

displaying explicitly the spinor indices $\alpha, \beta$. Note that the overall sign of the right-hand side (RHS) of Eqs. (17) and (18) differs from that in Ref. [8] since the definition of our Green's functions $G(x, y)$ and $S(x, y)$ differ by an overall factor of $-i$.

In many extensions of the SM, one encounters both chiral and Majorana fermions, which carry no conserved charge. It is useful, therefore, to derive the analogous continuity equation for the axial current $j_{\mu 5}(x)=$ $\left\langle\bar{\psi}(x) \gamma_{\mu} \gamma_{5} \psi(x)\right\rangle$. Doing so involves multiplying the Schwinger-Dyson equations by $\gamma_{5}$, performing the trace, and taking the difference rather than the sum of the components involving $S^{>}(x, y)$ on the LHS. The result is: 


$$
\begin{aligned}
\frac{\partial n_{5}}{\partial X_{0}}+\nabla \cdot \mathbf{j}_{5}(X)= & 2 i m P(X) \\
& +\int d^{3} z \int_{-\infty}^{X_{0}} d z_{0} \operatorname{Tr}\left\{\left[\Sigma^{>}(X, z) S^{<}(z, X)\right.\right. \\
& +S^{>}(X, z) \Sigma^{<}(z, X) \\
& -S^{<}(X, z) \Sigma^{>}(z, X) \\
& \left.\left.-\Sigma^{<}(X, z) S^{>}(z, X)\right] \gamma_{5}\right\}
\end{aligned}
$$

where $P(x)=\left\langle\bar{\psi}(x) \gamma_{5} \psi(x)\right\rangle$ and $m$ is the fermion mass. In principle, one could evaluate $P(x)$ using path-ordered perturbation theory as outlined above.

\section{B. Power Counting of Physical Scales}

Evaluating the various terms in Eqs. (17) and (18) leads to a system of coupled quantum transport equations for the charges that ultimately determine $n_{L}(x)$. On the LHS of these equations, it is conventional to parametrize $\mathbf{j}=$ $-D(\nabla n)$, in terms of the diffusion coefficient $D$ (whose expressions we take from Ref. [13]). The RHS involves integrating the products of various Green's functions and self-energies over the entire history of the system. In practice, this integral depends on the various time and energy scales associated with nonequilibrium dynamics at finite temperature and density. Here, we observe that there exists a hierarchy among these scales that leads to a natural power counting in their ratios (generically denoted here as $\boldsymbol{\epsilon}$ ) and that provides for a systematic expansion of the RHS of the transport Eqs. (17), (18), and (20).

The changing geometry associated with the expanding region of broken symmetry and the spacetime variation of the Higgs vevs leads to a decoherence of states that have, initially, precise energy and momentum. The effect is analogous to the quantum mechanical evolution of a particle in a box of side $L$. If the value of $L$ is changed to $L+$ $\Delta L$ in some time interval $\Delta t$, a state that is initially a stationary state for the original box will become an admixture of the stationary states of new box. The shorter the interval $\Delta t$ or the greater the wave number $k$ of the initial state, the smaller the probability will be of finding the particle in the state with the same wave number in the new system. The time scale that characterizes this decoherence, $\tau_{d}$, is naturally given by $\tau_{d} \sim 1 / v k$, where $v=$ $\Delta L / \Delta t$ is the velocity of expansion of the box and $k=$ $p / \hbar$. In the present case, the relevant velocity is just $v_{w}$, the expanding bubble wall velocity, and the relevant effective wave number $k$ depends on $|\mathbf{k}|$ and the wall thickness, $L_{w}$. The smaller the velocity or the longer the wavelength, the more adiabatic the dynamics of the expanding bubble become and the longer the decoherence time. Equilibrium dynamics are approached in the adiabatic limit: $\tau_{d} \rightarrow \infty$. The need to employ the CTP formalism follows from being in a situation with $v_{w}>0$, or $\tau_{d}<\infty$.

A second time scale that one encounters in quantum transport at the phase boundary arises from the presence of degeneracies among states in the thermal bath that vanish in the $T \rightarrow 0$ limit. At finite $T$, for example, a single, on-shell fermion may be degenerate with another state involving an on-shell fermion-gluon pair - a situation that is forbidden at $T=0$. Interactions of strength $g$ that cause mixing between such degenerate states give rise to thermal-or plasma-widths $\Gamma_{p}$ of order $\alpha T$ with $\alpha=$ $g^{2} / 4 \pi$, and transitions between the degenerate states take place on a plasma time scale $\tau_{p}$ of order $\sim 1 / \Gamma_{p}$. Again, the use of the CTP formalism is necessitated when $\tau_{p}<\infty$ or $T>0$.

A third time scale, which we denote $\tau_{\text {int }}$, is associated with the intrinsic frequency $\omega_{k}$ of the quasiparticle states that characterize the plasma dynamics. This time scale is naturally given by $\tau_{\text {int }} \sim 1 / \omega_{k}$. In the present case, we note that although the decoherence and plasma times are finite, they are typically much larger than $\tau_{\text {int }}$. For example, $\tau_{\text {int }} / \tau_{d}=v_{w} k / \omega_{k} \leq v_{w} / c$. Numerical studies indicate that $v_{w} / c \ll 1$. Similarly, $\tau_{\text {int }} / \tau_{p}=\alpha T / \omega_{k}$. Since quasiparticle thermal masses are of order $g T$ or larger, one also has that the latter ratio is smaller than unity. Thus, one is naturally led to expand the RHS of the transport equations in these ratios:

$$
\begin{aligned}
& 0<\tau_{\text {int }} / \tau_{d} \ll 1 \\
& 0<\tau_{\text {int }} / \tau_{p} \ll 1 .
\end{aligned}
$$

Finally, we observe that the generation of baryon number takes place in an environment of finite, but small particle number (or chiral charge) densities $n_{i}$ that are associated with chemical potentials $\mu_{i}$. For the temperatures and densities of interest here, one has $\left|\mu_{i}\right| / T \ll 1$, so that the latter ratio also provides for a natural expansion parameter. Denoting each of the ratios ${ }^{3}$ in Eq. (21) and $\mu_{i} / T$ by $\epsilon$, we show below that both the $C P$-violating sources and the relaxation term first arise at $\mathcal{O}\left(\epsilon^{2}\right)$, and we truncate our analysis at this order. We note that doing so introduces some simplifications into the evaluation of the RHS of the transport equations. For example, both the selfenergies $\Sigma^{\gtrless}$ and the Green's functions $G^{\gtrless}$, etc., depend on thermal distribution functions $f\left(T, \mu_{i}\right)$ that differ, in general, from their equilibrium values, $f_{0}\left(T, \mu_{i}\right)$. The difference $\delta f \equiv f\left(T, \mu_{i}\right)-f_{0}\left(T, \mu_{i}\right)$ that characterizes the departure from equilibrium will be at least of $\mathcal{O}(\epsilon)$, since it must vanish in the $v_{w} \rightarrow 0$ limit. We find below that the effect of having $\delta f \neq 0$ contributes at higher-order in $\epsilon$ than we consider here, so that we may use the equilibrium distribution functions in the Green's functions and selfenergies.

\footnotetext{
${ }^{3}$ For our purposes, it is not necessary to distinguish a hierarchy among the different scale ratios, as we work to leading nontrivial order in $\epsilon$.
} 


\section{Green's Functions at Nonzero Temperature and Density}

The computation of the various components of $\tilde{G}(x, y)$ and $\tilde{\Sigma}(x, y)$ appearing in Eqs. (17) and (18) at nonzero temperature and density requires knowledge of $\left(T, \mu_{i}\right)$-dependent fermion and boson propagators. The $T$-dependence of propagators has been studied extensively (see, e.g., Ref. [14] and references therein), while the $\mu_{i}$-dependence of fermion propagators has been studied in Refs. [15]. Here we summarize the features of $\left(T, \mu_{i}\right)$-dependent propagators which are important for our subsequent application of the real-time, CTP formalism of Sec. II, and give some more technical details in Appendix A.

For pedagogical purposes, we provide here a brief derivation of the noninteracting fermion propagator but only give final results for the case of interacting fermions and bosons. To do so, we start from the mode expansions for the field operators appearing in the free Dirac Lagrangian, $\psi(x)$ and $\bar{\psi}(x)$

$$
\begin{aligned}
\psi(x)= & \int \frac{d^{3} k}{(2 \pi)^{3}} \frac{1}{2 \omega_{\mathbf{k}}} \sum_{\alpha=1,2}\left[b_{\alpha}(\mathbf{k}) u(\mathbf{k}, \alpha) e^{-i k \cdot x}\right. \\
& \left.+d_{\alpha}^{\dagger}(\mathbf{k}) v(\mathbf{k}, \alpha) e^{i k \cdot x}\right] \\
\bar{\psi}(x)= & \int \frac{d^{3} k}{(2 \pi)^{3}} \frac{1}{2 \omega_{\mathbf{k}}} \sum_{\alpha=1,2}\left[b_{\alpha}^{\dagger}(\mathbf{k}) \bar{u}(\mathbf{k}, \alpha) e^{i k \cdot x}\right. \\
& \left.+d_{\alpha}(\mathbf{k}) \bar{v}(\mathbf{k}, \alpha) e^{-i k \cdot x}\right]
\end{aligned}
$$

where $k^{\mu}=\left(\omega_{\mathbf{k}}, \mathbf{k}\right), \omega_{\mathbf{k}}=\sqrt{|\mathbf{k}|^{2}+m^{2}}$, the mode operators satisfy:

$$
\begin{aligned}
\left\{b_{\alpha}(\mathbf{k}), b_{\beta}^{\dagger}\left(\mathbf{k}^{\prime}\right)\right\} & =\left\{d_{\alpha}(\mathbf{k}), d_{\beta}^{\dagger}\left(\mathbf{k}^{\prime}\right)\right\} \\
& =(2 \pi)^{3} \delta^{(3)}\left(\mathbf{k}-\mathbf{k}^{\prime}\right) 2 \omega_{\mathbf{k}} \delta_{\alpha \beta},
\end{aligned}
$$

and

$$
\begin{aligned}
& \left\langle b_{\alpha}(\mathbf{k})^{\dagger} b_{\beta}\left(\mathbf{k}^{\prime}\right)\right\rangle=f\left(\omega_{k}, \mu_{i}\right)(2 \pi)^{3} \delta^{(3)}\left(\mathbf{k}-\mathbf{k}^{\prime}\right) 2 \omega_{\mathbf{k}} \delta_{\alpha \beta} \\
& \left\langle d_{\alpha}(\mathbf{k})^{\dagger} d_{\beta}\left(\mathbf{k}^{\prime}\right)\right\rangle=f\left(\omega_{k},-\mu_{i}\right)(2 \pi)^{3} \delta^{(3)}\left(\mathbf{k}-\mathbf{k}^{\prime}\right) 2 \omega_{\mathbf{k}} \delta_{\alpha \beta},
\end{aligned}
$$

with $f\left(\omega, \mu_{i}\right)$ being the nonequilibrium Fermi distribution function. For our purposes, the relative change $\delta f\left(\omega, \mu_{i}\right) / f_{0}\left(\omega, \mu_{i}\right)$ enters the transport equations multiplying explicit factors of $\Gamma_{p}$ and either $v_{w}$ or $\mu$, so that in working to second order in $\epsilon$ we may replace $f$ by the equilibrium distributions $f_{0}\left(\omega, \mu_{i}\right)=n_{F}\left(\omega-\mu_{i}\right)=$ $\left[e^{\left(\omega-\mu_{i}\right) / T}+1\right]^{-1}$. Using the mode expansion (22) it is straightforward to show that $S^{>}(x, y)=\langle\psi(x) \bar{\psi}(y)\rangle$ and $S^{<}(x, y)=-\langle\bar{\psi}(y) \psi(x)\rangle$ can be expressed as:

$$
S^{\lambda}(x, y)=\int \frac{d^{4} k}{(2 \pi)^{4}} e^{-i k \cdot(x-y)} g_{F}^{\lambda}\left(k_{0}, \mu_{i}\right) \rho\left(k_{0}, \mathbf{k}\right)(k+m),
$$

( $\lambda$ denotes either " $>$ " or " $<$ ") in terms of the free particle spectral density:

$$
\begin{aligned}
\rho\left(k_{0}, \mathbf{k}\right)= & \frac{i}{2 \omega_{k}}\left[\left(\frac{1}{k_{0}-\omega_{k}+i \epsilon}-\frac{1}{k_{0}+\omega_{k}+i \epsilon}\right)\right. \\
& \left.-\left(\frac{1}{k_{0}-\omega_{k}-i \epsilon}-\frac{1}{k_{0}+\omega_{k}-i \epsilon}\right)\right],
\end{aligned}
$$

and the functions:

$$
\begin{aligned}
& g_{F}^{>}\left(k_{0}, \mu_{i}\right)=1-n_{F}\left(k_{0}-\mu_{i}\right) \\
& g_{F}^{<}\left(k_{0}, \mu_{i}\right)=-n_{F}\left(k_{0}-\mu_{i}\right) .
\end{aligned}
$$

The propagators $S^{t, \bar{t}}(x, y)$ can now be constructed from the $S^{\lambda}(x, y)$ as in Eqs. (8).

In the presence of interactions (characterized by a generic coupling $g$ ), the fermion propagator becomes considerably more complicated than given by Eq. (25). In particular, single fermion states can mix with other multiparticle states in the thermal bath, leading to the presence of additional poles (the "hole" modes) in the fermion propagator $[16,17]$. The general structure of the fermion propagator arising from these effects has been studied extensively at zero density [18]. In Appendix A we generalize to the case of nonzero $\mu_{i}$. For massless fermions, the resulting propagators are given by:

$$
\begin{aligned}
S^{\lambda}\left(x, y ; \mu_{i}\right)= & \int \frac{d^{4} k}{(2 \pi)^{4}} e^{-i k \cdot(x-y)} g_{F}^{\lambda}\left(k_{0}, \mu\right) \\
& \times\left[\frac{\gamma_{0}-\boldsymbol{\gamma} \cdot \hat{\mathbf{k}}}{2} \rho_{+}\left(k_{0}, \mathbf{k}, \mu_{i}\right)\right. \\
& \left.+\frac{\gamma_{0}+\boldsymbol{\gamma} \cdot \hat{\mathbf{k}}}{2} \rho_{-}\left(k_{0}, \mathbf{k}, \mu_{i}\right)\right],
\end{aligned}
$$

where $\hat{\mathbf{k}}$ is the unit vector in the $\mathbf{k}$ direction, and

$$
\begin{aligned}
\rho_{+}\left(k_{0}, \mathbf{k}, \mu_{i}\right)= & i\left[\frac{Z_{p}\left(k, \mu_{i}\right)}{k_{0}-\mathcal{E}_{p}\left(k, \mu_{i}\right)}-\frac{Z_{p}\left(k, \mu_{i}\right)^{*}}{k_{0}-\mathcal{E}_{p}\left(k, \mu_{i}\right)^{*}}\right. \\
& +\frac{Z_{h}\left(k,-\mu_{i}\right)^{*}}{k_{0}+\mathcal{E}_{h}\left(k,-\mu_{i}\right)^{*}}-\frac{Z_{h}\left(k,-\mu_{i}\right)}{k_{0}+\mathcal{E}_{h}\left(k,-\mu_{i}\right)} \\
& \left.+F\left(k_{0}^{*}, k, \mu_{i}\right)^{*}-F\left(k_{0}, k, \mu_{i}\right)\right]
\end{aligned}
$$

and

$$
\rho_{-}\left(k_{0}, \mathbf{k}, \mu_{i}\right)=\left[\rho_{+}\left(-k_{0}^{*}, \mathbf{k},-\mu_{i}\right)\right]^{*} .
$$

Here, $\mathcal{E}_{p}\left(k, \mu_{i}\right)$ and $-\mathcal{E}_{h}\left(k,-\mu_{i}\right)^{*}$ are the two (complex) roots (in $k_{0}$ ) of the equation:

$$
0=k_{0}-k+D_{+}\left(k_{0}, k, \mu_{i}\right)+i \epsilon
$$

where $i D_{ \pm}\left(k_{0}, k, \mu_{i}\right)$ are contributions to the inverse, re- 
tarded propagator proportional to $\left(\gamma_{0} \mp \boldsymbol{\gamma} \cdot \hat{\mathbf{k}}\right) / 2$ arising from interactions. The function $F\left(k_{0}, k, \mu_{i}\right)$ gives the nonpole part of the propagator, and $k=|\mathbf{k}|$. We find that the resonant contributions to the particle number-changing sources arise from the pole parts of the propagators, so from here on we neglect the terms containing $F\left(k_{0}, k, \mu_{i}\right)$.

In the limit $g \rightarrow 0$, one has $Z_{h} \rightarrow 0$ and $Z_{p} \rightarrow 1$, recovering the form of the propagator given in Eq. (25). For nonzero $g$, however, $Z_{h}$ is not of order $g^{2}$ since the particle and hole modes arise from mixtures of degenerate states. In particular, at $k=0$ one has $Z_{p}=Z_{h}=1 / 2$. As $k$ becomes large (of order the thermal mass or larger), $Z_{h} / Z_{p} \ll 1$, and the particle dispersion relation is well-approximated by $\mathcal{E}_{p}^{2}=|\mathbf{k}|^{2}+m^{2}\left(T, \mu_{i}\right)$, where $m\left(T, \mu_{i}\right)$ is the thermal mass. In our particular application to the MSSM, the gaugino $M_{i}$ masses will typically be taken to be of order several hundred $\mathrm{GeV}$, and for the $\mathrm{SU}(2)_{L} \times \mathrm{U}(1)_{Y}$ sector, thermal effects do not induce substantial mass corrections. We find that the gaugino contributions to the RHS of Eqs. (17) and (18) are dominated by momenta of order $M_{i}$, so that the hole contributions to the gaugino $S^{\lambda}(x, y)$ can be neglected. In contrast, for quarks we find nonnegligible contributions from the low-momentum region, so we retain the full structure given by Eqs. (28)-(30) in computing their contributions.

It has been noted in previous studies of quark damping rates that the one-loop thermal widths $\Gamma_{p, h}=\operatorname{Im} \mathcal{E}_{p, h}(k, \mu)$ are gauge-dependent (see Ref. [19] and Ref. [3] therein), whereas the thermal masses $m(T, \mu)$ entering $\mathcal{E}_{p, h}$ are gauge-independent to this order. Gauge-independent widths can be obtained by performing an appropriate resummation of hard thermal loops (HTLs) $[14,19,20]$. The latter are associated with momenta $k_{0}, k \sim g T$, for which the one-loop functions $D_{ \pm}\left(k_{0}, k, \mu\right)$ are of the same order in $g$ as the tree-level inverse propagators. In what follows, we will estimate the widths $\Gamma_{p, h}$ based on existing computations of damping [21-23], deferring a complete computation of the gauge-invariant, $\mu_{i}$-dependent contributions in the MSSM to a future study. In general, the residues $Z_{p, h}$ also carry a gauge-dependence, and at this time we are not aware of any HTL resummation that could eliminate this dependence. In principle, elimination of this gaugedependence requires inclusion of one-loop vertex corrections in the computation of the $\Sigma^{\lambda}(x, y)$ and $S^{\lambda}(x, y)$ appearing on the RHS of Eqs. (17) and (18), and we again defer a complete one-loop computation to a future study.

The derivation of the finite-density scalar propagators proceeds along similar lines. Starting from the mode expansion of the free scalar field $\phi(x)$ in terms of plane-wave solutions to the Klein-Gordon equation and following analogous arguments as for fermions, one arrives at the following scalar Green's functions:

$$
G^{\lambda}(x, y)=\int \frac{d^{4} k}{(2 \pi)^{4}} e^{-i k \cdot(x-y)} g_{B}^{\lambda}\left(k_{0}, \mu_{i}\right) \rho\left(k_{0}, \mathbf{k}\right)
$$

where the equilibrium distribution functions are:

$$
\begin{aligned}
& g_{B}^{>}(\omega, \mu)=1+n_{B}\left(\omega-\mu_{i}\right) \\
& g_{B}^{<}(\omega, \mu)=n_{B}\left(\omega-\mu_{i}\right),
\end{aligned}
$$

with $n_{B}(x)=1 /\left(e^{x / T}-1\right)$ and $\rho\left(k_{0}, \mathbf{k}\right)$ given by Eq. (26). As with fermions, one may include the effect of thermal masses and widths by replacing $m^{2} \rightarrow m^{2}\left(T, \mu_{i}\right)$ and $i \epsilon \rightarrow$ $i \epsilon+i \Gamma\left(T, \mu_{i}\right)$.

\section{SOURCE TERMS FOR QUANTUM TRANSPORT}

The expressions for $G^{\lambda}(x, y)$ and $S^{\lambda}(x, y)$ now allow us to compute the perturbative contributions to the source terms on the RHS of Eqs. (17) and (18) starting from a given electroweak model Lagrangian. Here, we work within the MSSM as an illustrative case, but emphasize that the methods are general. The Feynman rules giving the relevant interaction vertices in the MSSM are taken from Ref. [24], and in what follows, we only write down those relevant for the computations undertaken here. It is useful, however, to place our calculation in a broader context by considering the various classes of graphs that generate different terms in the QTEs. The simplest topologies are those involving scattering of particles and their superpartners from the spacetime varying Higgs vevs (generically denoted $v$ ) in the plasma [Fig. 1]. These graphs give rise to both the $C P$-violating source terms discussed in Ref. [8] as well as terms proportional to chiral charge. The latter involve the number densities of at most two different species, such as the left- and right-handed top quarks [Fig. 1(a)] or their superpartners [Fig. 1(b)]. For purposes of illustration, we follow Ref. [8] and work in a basis of mass eigenstates in the unbroken phase, treating the interactions with the Higgs vevs perturbatively. This approximation should be reasonable near the phase transition boundary, where both the vevs and their rate of change are small, but it clearly breaks down farther inside the bubble wall, where the vevs become large (of order the phase transition temperature, $T_{c}$ ). In general, one would like to perform a resummation to all orders in the vevs, possibly employing the approximation scheme proposed in

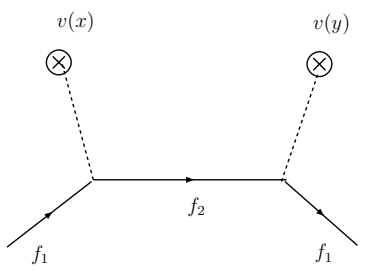

(a)

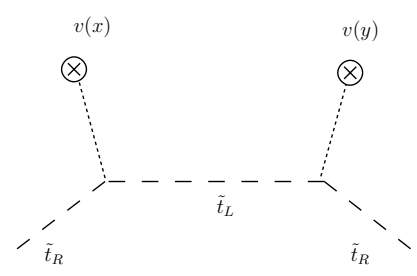

(b)
FIG. 1. Contributions to the relevant self-energies from scattering of particles from the spacetime varying Higgs vevs. 

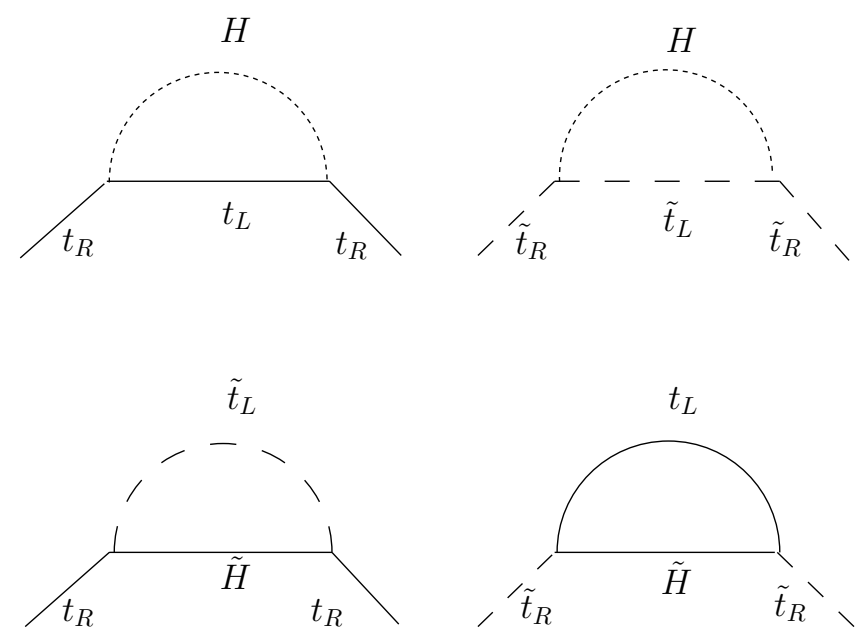

FIG. 2. Contributions to the relevant self-energies from Yukawa interactions.

Refs. $[11,25]$. We postpone a treatment of this resummation to a future study. ${ }^{4}$

Yukawa interactions involving quarks (squarks) and Higgs (Higgsinos) are illustrated in Fig. 2 (the selfenergies $\Sigma^{\lambda}(x, y)$ are obtained by amputating the external legs). These interactions cause transitions such as $f \leftrightarrow f H$, $\tilde{f} \leftrightarrow \tilde{f} H$, and $f \leftrightarrow \tilde{f} \tilde{H}$. Contributions from gauge interactions appear in Fig. 3. The latter induce transitions of the type $f \leftrightarrow f V, \tilde{f} \leftrightarrow \tilde{f} V$, and $f \leftrightarrow \tilde{f} \tilde{V}$. In general, one expects the Yukawa and gauge interactions involving three different species to depend on sums and differences of the corresponding chemical potentials, as in $\mu_{f}-\mu_{\tilde{f}}-\mu_{\tilde{V}}$ for the supergauge interactions. In previous studies, it has been assumed that the gauginos $\tilde{V}$ are sufficiently light and the coefficients of the corresponding terms in the QTEs sufficiently large than one has $\mu_{\tilde{V}} \approx 0$ and $\mu_{f} \approx \mu_{\tilde{f}}$. Although the quantitative validity of this assumption could be explored using our framework here, we defer that analysis to a future study and take $\mu_{\tilde{V}} \approx 0, \mu_{f} \approx \mu_{\tilde{f}}$. Consequently, one may, as in Ref. [10], define a common chemical potential for SM particles (including the two Higgs doublets) and their superpartners.

In previous studies, it has also been assumed-based largely on simple estimates (see, e.g., Ref. [10]) — that the Yukawa interactions of Fig. 2 are sufficiently fast that they decouple from the set of QTEs, leading to relations between the chemical potentials for the Higgs (Higgsino) fields and those for matter fields. For example, Yukawa interactions that couple the Higgs doublet fields $H$ with those of the third generation $\mathrm{SU}(2)_{L}$ doublet quarks, $Q$

\footnotetext{
${ }^{4}$ The authors of Ref. [11] find that carrying out such a resummation reduces the resonant enhancements of the $C P$-violating sources, but they did not consider the $C P$-conserving, chirality-changing terms that are our focus here. The consistency of the proposed approximate resummation with our power counting remains to be analyzed.
}
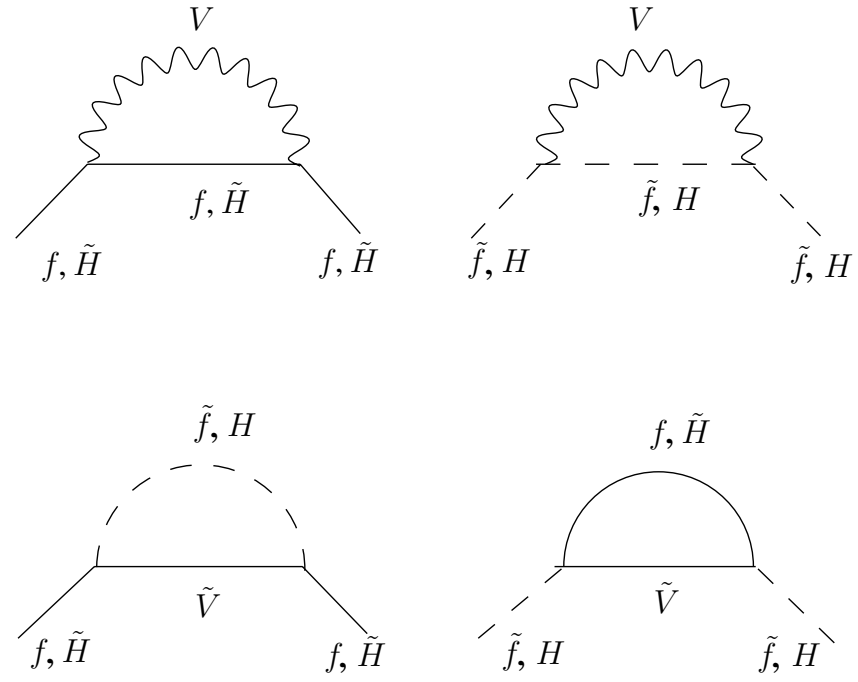

FIG. 3. Representative contributions to self-energies from (super)gauge interactions.

with the singlet top quark supermultiplet field, $T$, generate terms of the form:

$$
\Gamma_{Y}\left(\mu_{Q}-\mu_{T}+\mu_{H}\right) .
$$

To the extent that $\Gamma_{Y}$ is much larger than the other transport coefficients appearing in Eqs. (17) and (18), one has $\mu_{Q}=$ $\mu_{T}-\mu_{H}$ plus terms of $\mathcal{O}\left(1 / \Gamma_{Y}\right)$. The remaining terms in the QTEs will involve the $C P$-violating sources, sphaleron terms, and terms that couple left- and right-handed chiral charges, such as $\Gamma_{M}\left(\mu_{Q}-\mu_{T}\right)$. Again, this assumption could be tested using the current framework, but the computation of $\Gamma_{Y}$ is considerably more arduous than those discussed below, where we focus on the $C P$-violating sources and the $\Gamma_{M}$-type terms that are generated by the diagrams in Fig. 1.

\section{A. Bosons}

We consider first the scalar interactions in Fig. 1(a). The largest contributions involve the $\mathrm{L}$ and $\mathrm{R}$ top squarks, $\tilde{t}_{L, R}$ owing to their large Yukawa coupling, $y_{t}$. In the basis of weak eigenstates, the relevant interaction Lagrangian is:

$$
\mathcal{L}=y_{t} \tilde{t}_{L} \tilde{t}_{R}^{*}\left(A_{t} v_{u}-\mu^{*} v_{d}\right)+\text { H.c., }
$$

where $v_{u, d}$ are the vevs of $H_{u, d}^{0}$, and we take $v \equiv \sqrt{v_{u}^{2}+v_{d}^{2}}$ and $\tan \beta \equiv v_{u} / v_{d}$. Note that in Eq. (35) we allow the $v_{u, d}$ to be spacetime-dependent. In the region of broken electroweak symmetry and stable vevs, we have $m_{t}=y_{t} v_{u}$.

Using the Feynman rules for path ordered perturbation theory, it is straightforward to show that the diagrams in Fig. 1(a) generate contributions to $\tilde{\Sigma}_{R}(x, y)$ of the form:

$$
\tilde{\Sigma}_{R}(x, y)=-g(x, y) \tilde{G}_{L}^{0}(x, y),
$$

where 


$$
g(x, y)=y_{t}^{2}\left[A_{t} v_{u}(x)-\mu^{*} v_{d}(x)\right]\left[A_{t}^{*} v_{u}(y)-\mu v_{d}(y)\right] .
$$

Substituting Eq. (36) into Eq. (17) leads to:

$$
\partial_{\mu} \tilde{t}_{R}^{\mu}(x)=S_{\tilde{t}_{R}}(x)
$$

for right-handed top squarks, where $\tilde{t}_{R}^{\mu}$ is the corresponding current density and the source $S_{\tilde{t}_{R}}(x)$ is

$$
\begin{aligned}
S_{\tilde{t}_{R}}(x)= & -\int d^{3} z \int_{-\infty}^{x_{0}} d z_{0}\{[g(x, z)+g(z, x)] \\
& \times \operatorname{Re}\left[G_{L}^{>}(x, z) G_{R}^{<}(z, x)-G_{L}^{<}(x, z) G_{R}^{>}(z, x)\right] \\
& +i[g(x, z)-g(z, x)] \\
& \left.\times \operatorname{Im}\left[G_{L}^{>}(x, z) G_{R}^{<}(z, x)-G_{L}^{<}(x, z) G_{R}^{>}(z, x)\right]\right\},
\end{aligned}
$$

where the $L, R$ subscripts indicate the propagators for the $\mathrm{L}$ and $\mathrm{R}$ top squarks.

The first term in the integrand of $S_{\tilde{t}_{R}}(x)$ is $C P$-conserving and leads to the $\Gamma_{M}$-type terms discussed above, while the second term in the integrand provides the $C P$-violating sources. We concentrate first on the former. Expanding $g(x, z)$ about $z=x$ it is straightforward to show that only terms involving even powers of derivatives survive in $g(x, z)+g(z, x)$. Under the assumptions of gentle spacetime dependence of the $v_{i}(x)$ near the phase boundary, we will neglect terms beyond leading order and take $g(x, z)+$ $g(z, x) \approx 2 g(x, x)$. Consequently, the $C P$-conserving source is:

$$
\begin{aligned}
S_{\tilde{t}_{R}}^{C P}(x) \approx & -2 g(x, x) \operatorname{Re} \int d^{3} z \int_{-\infty}^{x_{0}} d z_{0}\left[G_{L}^{>}(x, z) G_{R}^{<}(z, x)-G_{L}^{<}(x, z) G_{R}^{>}(z, x)\right] \\
= & -2 g(x, x) \operatorname{Re} \int d^{3} z \int_{-\infty}^{x_{0}} d z_{0} \int \frac{d^{4} k}{(2 \pi)^{4}} \int \frac{d^{4} q}{(2 \pi)^{4}} e^{-i(k-q) \cdot(x-z)} \rho_{L}\left(k_{0}, \mathbf{k}\right) \rho_{R}\left(q_{0}, \mathbf{q}\right)\left[g_{B}^{>}\left(k_{0}, \mu_{L}\right) g_{B}^{<}\left(q_{0}, \mu_{R}\right)\right. \\
& \left.-g_{B}^{<}\left(k_{0}, \mu_{L}\right) g_{B}^{>}\left(q_{0}, \mu_{R}\right)\right],
\end{aligned}
$$

with

$$
g(x, x)=y_{t}^{2}\left[|\mu|^{2} v_{d}^{2}(x)+\left|A_{t}\right|^{2} v_{u}^{2}(x)-2 v_{d}(x) v_{u}(x) \operatorname{Re}\left(\mu A_{t}\right)\right] .
$$

Performing the $d^{3} z$ integral leads to a $\delta$ function in momentum space. After carrying out the $d^{3} q$ integral, we perform the contour integrals for $k_{0}$ and $q_{0}$, expand to first order in $\mu_{L, R} / T$, and obtain:

$$
S_{\tilde{t}_{R}}^{C P}(x)=-\frac{1}{T} \frac{N_{C} y_{t}^{2}}{2 \pi^{2}}\left|A_{t} v_{u}(x)-\mu^{*} v_{d}(x)\right|^{2} \int_{0}^{\infty} \frac{d k k^{2}}{\omega_{L} \omega_{R}} \operatorname{Im}\left\{\frac{\mu_{L} h_{B}\left(\mathcal{E}_{L}\right)-\mu_{R} h_{B}\left(\mathcal{E}_{R}^{*}\right)}{\mathcal{E}_{L}-\mathcal{E}_{R}^{*}}+\frac{\mu_{R} h_{B}\left(\mathcal{E}_{R}\right)-\mu_{L} h_{B}\left(\mathcal{E}_{L}\right)}{\mathcal{E}_{L}+\mathcal{E}_{R}}\right\},
$$

where

$$
\begin{aligned}
\omega_{L, R}^{2} & =|\mathbf{k}|^{2}+M_{\tilde{t}_{L, R}}^{2} \\
\mathcal{E}_{L, R} & =\omega_{L, R}-i \Gamma_{L, R} \\
h_{B}(x) & =-\frac{e^{x / T}}{\left(e^{x / T}-1\right)^{2}},
\end{aligned}
$$

and $M_{\tilde{t}_{L, R}}, \Gamma_{L, R}$ are the thermal masses and widths for the $\tilde{t}_{L, R}$, and the factor of $N_{C}$ comes from summing over the colors. Note that, in arriving at Eq. (42), we have neglected the $\mu_{i}$-dependence of the pole residues $Z\left(T, \mu_{L, R}\right)$, thermal frequencies, $\omega_{L, R}\left(T, \mu_{L, R}\right)$, and widths, $\Gamma_{L, R}\left(T, \mu_{L, R}\right)$. The effect on $S_{\tilde{t}_{R}}^{C P}(x)$ of the $\mu_{i}$-dependence of the residues and thermal frequencies is subleading in the gauge and Yukawa couplings, whereas the effect from the thermal widths occurs at leading order. The $\mu_{i}$-dependence of $\Gamma_{L, R}\left(T, \mu_{L, R}\right)$ is simply not known, however, so we do not include it here. A more explicit expression for the dependence of $S_{\tilde{t}_{R}}^{C P}(x)$ on the thermal frequencies and widths is given in Eqs. (B1)-(B3) Appendix B.

For purposes of future analysis, it is useful to rewrite Eq. (42) as:

$$
S_{\tilde{t}_{R}}^{C P}=\Gamma_{\tilde{t}}^{+}\left(\mu_{L}+\mu_{R}\right)+\Gamma_{\tilde{t}}^{-}\left(\mu_{L}-\mu_{R}\right)
$$

where

$$
\begin{aligned}
\Gamma_{\tilde{t}}^{ \pm}= & -\frac{1}{T} \frac{N_{C} y_{t}^{2}}{4 \pi^{2}}\left|A_{t} v_{u}(x)-\mu^{*} v_{d}(x)\right|^{2} \\
& \times \int_{0}^{\infty} \frac{d k k^{2}}{\omega_{R} \omega_{L}} \operatorname{Im}\left\{\frac{h_{B}\left(\mathcal{E}_{L}\right) \mp h_{B}\left(\mathcal{E}_{R}^{*}\right)}{\mathcal{E}_{L}-\mathcal{E}_{R}^{*}}\right. \\
& \left.-\frac{h_{B}\left(\mathcal{E}_{L}\right) \mp h_{B}\left(\mathcal{E}_{R}\right)}{\mathcal{E}_{L}+\mathcal{E}_{R}}\right\} .
\end{aligned}
$$

Before proceeding with the $C P$-violating source, we comment briefly on the structure of Eqs. (44) and (45). In particular, we note that

(i) Terms of the type $\Gamma_{\tilde{t}}^{+}$are absent from the conventional QTEs for EWB. It is straightforward to see that in the absence of interactions that distinguish between $\tilde{t}_{L}$ and $\tilde{t}_{R}, \Gamma_{\tilde{t}}^{+}=0$, as the integrand of Eq. (45) is antisymmetric under $L \leftrightarrow R$ interchange. In contrast, the transport coefficient $\Gamma_{\tilde{t}}^{-}$is nonzero in the limit of exact $\tilde{t}_{L} \leftrightarrow \tilde{t}_{R}$ symmetry. This term corresponds to the usual damping term in 
the QTEs associated with scattering from the Higgs vevs.

(ii) In the absence of thermal widths $\Gamma_{L, R}$, the quantity in brackets in Eq. (45) is purely real, and so the damping term would be zero.

(iii) The structure of the energy denominators implies a resonant enhancement of the integrand for $M_{\tilde{t}_{L}}^{2} \sim$ $M_{\tilde{t}_{R}}^{2}$. A similar effect was observed to occur for the $C P$-violating sources (see below) in Refs. $[8,26]$. The expression in Eq. (45) makes it clear that the relaxation terms display a resonant behavior as well. The resulting quantitative impact of this resonance on the baryon asymmetry is discussed in Sect. V.

Properties (ii) and (iii) are shared by all source and damping terms, we discuss below. Note that the explicit factors of $\mu_{L, R} / T$ and property (ii) imply that, away from the resonance region, $S_{\tilde{t}_{R}}^{C P}$ is $\mathcal{O}\left(\epsilon^{2}\right)$.

The computation of the $C P$-violating source, given by the second term in Eq. (39), proceeds along similar lines. In this case, the coefficient $[g(x, z)-g(z, x)]$ vanishes for $x=z$, so we must retain terms at least to first order in the expansion about $x=z$ :

$$
\begin{aligned}
g(x, z)-g(z, x)= & 2 i y_{t}^{2} \operatorname{Im}\left(\mu A_{t}\right)\left[v_{d}(x) v_{u}(z)-v_{d}(z) v_{u}(x)\right] \\
= & 2 i y_{t}^{2} \operatorname{Im}\left(\mu A_{t}\right)(z-x)^{\lambda}\left[v_{d}(x) \partial_{\lambda} v_{u}(x)\right. \\
& \left.-v_{u}(x) \partial_{\lambda} v_{d}(x)\right]+\cdots,
\end{aligned}
$$

where the $+\cdots$ indicate higher order terms in the derivative expansion that we neglect for the same reasons as discussed previously. When the linear term in Eq. (46) is substituted in Eq. (39), only the time component yields a nonzero contribution. The spatial components vanish due to the spatial isotropy of the spectral density: $g_{B}^{\lambda}\left(k_{0}, \mu\right) \rho\left(k_{0},|\mathbf{k}|\right) \equiv g_{B}^{\lambda}\left(k_{0}, \mu\right) \rho\left(k_{0}, \mathbf{k}\right)$. We may then make the replacement:

$$
\begin{aligned}
g(x, z)-g(z, x) \rightarrow & 2 i y_{t}^{2} \operatorname{Im}\left(\mu A_{t}\right)\left[v_{d}(x) \dot{v}_{u}(x)\right. \\
& \left.-\dot{v}_{d}(x) v_{u}(x)\right](z-x)^{0} \\
= & 2 i y_{t}^{2} \operatorname{Im}\left(\mu A_{t}\right) v(x)^{2} \dot{\beta}(x)(z-x)^{0} .
\end{aligned}
$$

In general, we expect $\dot{\beta}$ to be of order $v_{w} / c$, so that the $C P$-violating source is first order in one of the small expansion parameters discussed earlier. Consequently, when evaluating this term, we may neglect the $\mu_{L, R}$-dependence of the $g_{B}^{\lambda}\left(k_{0}, \mu\right)$. After carrying out the $\left(k_{0}, q_{0}\right)$ contour integrals and performing the time integration, we obtain:

$$
\begin{aligned}
S_{\tilde{t}_{R}}^{C P}= & \frac{N_{C} y_{t}^{2}}{2 \pi^{2}} \operatorname{Im}\left(\mu A_{t}\right) v(x)^{2} \dot{\beta}(x) \\
& \times \int_{0}^{\infty} \frac{d k k^{2}}{\omega_{R} \omega_{L}} \operatorname{Im}\left\{\frac{n_{B}\left(\mathcal{E}_{R}^{*}\right)-n_{B}\left(\mathcal{E}_{L}\right)}{\left(\mathcal{E}_{L}-\mathcal{E}_{R}^{*}\right)^{2}}\right. \\
& \left.+\frac{1+n_{B}\left(\mathcal{E}_{R}\right)+n_{B}\left(\mathcal{E}_{L}\right)}{\left(\mathcal{E}_{L}+\mathcal{E}_{R}\right)^{2}}\right\} .
\end{aligned}
$$

Again, property (ii), in conjunction with the factor of $\dot{\beta} \propto$ $v_{w}$, implies that $S_{\tilde{t}_{R}}^{C P}$ is $\mathcal{O}\left(\epsilon^{2}\right)$. An expression giving a more explicit dependence on the widths and frequencies appears in Eq. (B4) of Appendix B. We note that our result agrees with that of Ref. [8] except for a different relative sign in front of the $\cos 2 \phi$ term of that equation and the overall factor of $N_{C}$.

\section{B. Massive fermions}

The computations for fermions proceed along similar lines. We consider first the source terms for Higgsinos. We recall that it is useful to redefine the Higgsino fields to remove the complex phase from the Higgsino mass term:

$$
\begin{aligned}
\mathcal{L}_{\tilde{H}}^{\text {mass }}= & \mu\left(\psi_{H_{d}^{0}} \psi_{H_{u}^{0}}-\psi_{H_{d}^{-}} \psi_{H_{u}^{+}}\right) \\
& +\mu^{*}\left(\bar{\psi}_{H_{d}^{0}} \bar{\psi}_{H_{u}^{0}}-\bar{\psi}_{H_{d}^{-}} \bar{\psi}_{H_{u}^{+}}\right)
\end{aligned}
$$

via

$$
\psi_{H_{d}^{0,-}} \rightarrow \tilde{H}_{d}^{0,-}, \quad \psi_{H_{u}^{0,+}} \rightarrow e^{-i \phi_{\mu}} \tilde{H}_{u}^{0,+}
$$

leading to:

$$
\mathcal{L}_{\tilde{H}}^{\operatorname{mass}}=|\mu|\left(\tilde{H}_{u}^{0} \tilde{H}_{d}^{0}-\tilde{H}_{d}^{-} \tilde{H}_{u}^{+}\right)+|\mu|\left(\tilde{H}_{d}^{0 \dagger} \tilde{H}_{u}^{0 \dagger}-\tilde{H}_{d}^{-\dagger} \tilde{H}_{u}^{+\dagger}\right) .
$$

Defining the four component spinors,

$$
\Psi_{\tilde{H}^{+}}=\left(\begin{array}{c}
\tilde{H}_{u}^{+} \\
\tilde{H}_{d}^{-\dagger}
\end{array}\right), \quad \Psi_{\tilde{H}^{0}}=\left(\begin{array}{c}
-\tilde{H}_{u}^{0} \\
\tilde{H}_{d}^{0 \dagger}
\end{array}\right)
$$

for the Higgsinos, and

$$
\Psi_{\tilde{W}^{+}}=\left(\begin{array}{c}
\tilde{W}^{+} \\
\tilde{W}^{-\dagger}
\end{array}\right), \quad \Psi_{\tilde{W}^{0}}=\left(\begin{array}{c}
\tilde{W}^{3} \\
\tilde{W}^{3 \dagger}
\end{array}\right), \quad \Psi_{\tilde{B}}=\left(\begin{array}{c}
\tilde{B} \\
\tilde{B}^{\dagger}
\end{array}\right)
$$

for the gauginos, leads to the Higgsino-gaugino-vev interaction:

$$
\begin{aligned}
\mathcal{L}^{\mathrm{int}}= & -g_{2} \bar{\Psi}_{\tilde{H}^{+}}\left[v_{d}(x) P_{L}+v_{u}(x) e^{i \phi_{\mu}} P_{R}\right] \Psi_{\tilde{W}^{+}} \\
& -\frac{1}{\sqrt{2}} \bar{\Psi}_{\tilde{H}^{0}}\left[v_{d}(x) P_{L}+v_{u}(x) e^{i \phi_{\mu}} P_{R}\right]\left(g_{2} \Psi_{\tilde{W}^{0}}\right. \\
& \left.-g_{1} \Psi_{\tilde{B}}\right)+ \text { H.c. }
\end{aligned}
$$

Note that the spinors $\Psi_{\tilde{H}^{0}}$ and $\Psi_{\tilde{H}^{+}}$satisfy a Dirac equation with Dirac mass $|\mu|$, even though the $\tilde{H}_{d, u}^{0}$ are Majorana particles. The $\Psi_{\tilde{W}^{ \pm}}$are Dirac particles of mass 
$M_{2}$, whereas the $\Psi_{\tilde{W}^{0}}$ and $\Psi_{\tilde{B}^{0}}$ are Majorana particles with Majorana masses $M_{2}$ and $M_{1}$, respectively. We also note that the construction of the Dirac spinor $\Psi_{\tilde{H}^{0}}$ allows one to define a vector charge and corresponding chemical potential, $\mu_{\tilde{H}^{0}}$, for the neutral Higgsinos, even though they are Majorana particles. In contrast, there exists no such vector charge for the $\Psi_{\tilde{W}^{0}}$ and $\Psi_{\tilde{B}^{0}}$. One may, however, study the quantum transport of the axial charge of the Majorana fermions using Eq. (20). An attempt to do so for the neutral Higgsinos was made in Ref. [11], though only the $C P$-violating sources were evaluated using nonequilibrium methods. The impact of the corresponding axial charge density on the baryon asymmetry was found to be small. We return to this issue in a future study, and consider only the vector densities below.

The most straightforward computation is that of the $\tilde{H}^{ \pm}$ source terms. For notational convenience, we rewrite the chargino interactions in Eq. (54) as:

$$
-g_{2} \bar{\Psi}_{\tilde{H}^{+}}\left[g_{L}(x) P_{L}+g_{R}(x) P_{R}\right] \Psi_{\tilde{W}^{+}}+\text {H.c. }
$$

In this case, the self-energy generated by Fig. 1(a) is:

$$
\begin{aligned}
\tilde{\Sigma}_{\tilde{H}^{ \pm}}(x, y)= & -g_{2}^{2}\left[g_{L}(x) P_{L}+g_{R}(x) P_{R}\right] \tilde{S}_{\tilde{W}^{ \pm}}(x, y) \\
& \times\left[g_{L}(y)^{*} P_{R}+g_{R}(y)^{*} P_{L}\right] .
\end{aligned}
$$

Defining:

$$
\begin{aligned}
& g_{A}(x, y) \equiv \frac{g_{2}^{2}}{2}\left[g_{L}(x) g_{L}(y)^{*}+g_{R}(x) g_{R}(y)^{*}\right] \\
& g_{B}(x, y) \equiv \frac{g_{2}^{2}}{2}\left[g_{L}(x) g_{R}(y)^{*}+g_{R}(x) g_{L}(y)^{*}\right],
\end{aligned}
$$

we obtain for the RHS of Eq. (18):

$$
\begin{aligned}
S_{\tilde{H}^{ \pm}}(x)= & \int d^{3} z \int_{-\infty}^{x_{0}} d z_{0} \sum_{j=A, B}\left\{\left[g_{j}(x, z)+g_{j}(z, x)\right]\right. \\
& \times \operatorname{Re} \operatorname{Tr}\left[S_{\tilde{W}^{ \pm}}^{>}(x, z) S_{\tilde{H}^{ \pm}}^{<}(z, x)\right. \\
& \left.-S_{\tilde{W}^{ \pm}}^{<}(x, z) S_{\tilde{H}^{ \pm}}^{>}(z, x)\right]_{j}+i\left[g_{j}(x, z)-g_{j}(z, x)\right] \\
& \times \operatorname{Im} \operatorname{Tr}\left[S_{\tilde{W}^{ \pm}}^{>}(x, z) S_{\tilde{H}^{ \pm}}^{<}(z, x)\right. \\
& \left.\left.-S_{\tilde{W}^{ \pm}}^{<}(x, z) S_{\tilde{H}^{ \pm}}^{>}(z, x)\right]_{j}\right\},
\end{aligned}
$$

where the subscripts " $A$ " and " $B$ " on the traces denote the contributions arising from the $k$ and $m$ terms, respectively, in the spectral function in Eq. (25) (an overall factor of $1 / 2$ due to the presence of the chiral projectors $P_{L, R}$ has been absorbed in the definition of the $g_{A, B}$ ).

As in the case of the scalar fields, the leading densitydependent, $C P$-conserving contribution to $S_{\tilde{H}^{ \pm}}(x)$ arises from the term in Eq. (58) containing the $x \leftrightarrow z$ symmetric factors $\left[g_{j}(x, z)+g_{j}(z, x)\right]$. To lowest order in $\boldsymbol{v}_{w}$, we may set $x=z$ in these factors. Using the spectral representation of the $S^{\lambda}(x, y)$ given in Eq. (25), including gauge-invariant thermal masses and widths, and expanding to first order in $\mu_{i} / T$, we obtain the chirality-changing source term:

$$
S_{\tilde{H}^{ \pm}}^{C P}(x)=\Gamma_{\tilde{H}^{ \pm}}^{+}\left(\mu_{\tilde{W}^{ \pm}}+\mu_{\tilde{H}^{ \pm}}\right)+\Gamma_{\tilde{H}^{ \pm}}^{-}\left(\mu_{\tilde{W}^{ \pm}}-\mu_{\tilde{H}^{ \pm}}\right),
$$

where

$$
\begin{aligned}
\Gamma_{\tilde{H}^{ \pm}}^{ \pm}= & \frac{1}{T} \frac{g_{2}^{2}}{2 \pi^{2}} v(x)^{2} \int_{0}^{\infty} \frac{d k k^{2}}{\omega_{\tilde{H}} \omega_{\tilde{W}}} \operatorname{Im}\left\{\left[\mathcal{E}_{\tilde{W}} \mathcal{E}_{\tilde{H}}^{*}-k^{2}\right.\right. \\
& \left.+M_{2}|\mu| \cos \phi_{\mu} \sin 2 \beta\right] \frac{h_{F}\left(\mathcal{E}_{\tilde{W}}\right) \mp h_{F}\left(\mathcal{E}_{\tilde{H}}^{*}\right)}{\mathcal{E}_{\tilde{W}}-\mathcal{E}_{\tilde{H}}^{*}} \\
& +\left[\mathcal{E}_{\tilde{W}} \mathcal{E}_{\tilde{H}}+k^{2}-M_{2}|\mu| \cos \phi_{\mu} \sin 2 \beta\right] \\
& \left.\times \frac{h_{F}\left(\mathcal{E}_{\tilde{W}}\right) \mp h_{F}\left(\mathcal{E}_{\tilde{H}}\right)}{\mathcal{E}_{\tilde{W}}+\mathcal{E}_{\tilde{H}}}\right\},
\end{aligned}
$$

where the definitions of $\omega_{\tilde{H}, \tilde{W}}$ and $\mathcal{E}_{\tilde{H}, \tilde{W}}$ are analogous to those given in Eqs. (43) and

$$
h_{F}(x)=\frac{e^{x / T}}{\left(e^{x / T}+1\right)^{2}} .
$$

Also, the factor of $\cos \varphi_{\mu}$ is very nearly 1 for the region of small $\varphi_{\mu}$ in which we find ourselves in subsequent sections. The explicit dependence of $\Gamma_{\tilde{H}^{ \pm}}^{ \pm}$on thermal frequencies and widths is given in Eq. (B5) of Appendix B.

In the present case, we follow Ref. [10] and assume no net density of gauginos, thereby setting $\mu_{\tilde{W}^{ \pm}}=0$ in Eq. (59) and giving:

$$
S_{\tilde{H}^{ \pm}}^{C P}(x)=-\Gamma_{\tilde{H}^{ \pm}} \mu_{\tilde{H}^{ \pm}},
$$

with $\Gamma_{\tilde{H}^{ \pm}}=\Gamma_{\tilde{H}^{ \pm}}^{-}-\Gamma_{\tilde{H}^{ \pm}}^{+}$. In this case, it is straightforward to obtain the corresponding source term for the neutral Higgsinos,

$$
S_{\tilde{H}^{0}}^{C P}(x)=-\Gamma_{\tilde{H}^{0}} \mu_{\tilde{H}^{0}},
$$

where $\Gamma_{\tilde{H}^{0}}$ can be obtained from the formulae for $\Gamma_{\tilde{H}^{ \pm}}$by making the following replacements: $g_{2} \rightarrow g_{2} / \sqrt{2}$ for $\tilde{W}^{0}$ intermediate states and $g_{2} \rightarrow g_{1} / \sqrt{2}, \omega_{\tilde{W}} \rightarrow \omega_{\tilde{B}}$, and $\Gamma_{\tilde{W}} \rightarrow \Gamma_{\tilde{B}}$ for the $\tilde{B}$ intermediate states.

The Higgsino $C P$-violating source arises from the second term in Eq. (58). As before, we expand the $g_{j}(x, z)$ to first order about $x=z$ and observe that only the $x_{0}-z_{0}$ component survives when the $d^{3} z$ integration is performed. Also note that $g_{A}(x, z)-g_{A}(z, x)=2 i \operatorname{Im} g_{A}(x, z)=0$ so that only the terms proportional to the Higgsino and gaugino masses contribute. The result is:

$$
\begin{aligned}
S_{\tilde{H}^{ \pm}}^{C P}(x)= & \frac{g_{2}^{2}}{\pi^{2}} v(x)^{2} \dot{\beta}(x) M_{2}|\mu| \sin \phi_{\mu} \int_{0}^{\infty} \frac{d k k^{2}}{\omega_{\tilde{H}} \omega_{\tilde{W}}} \\
& \times\left\{\frac{n_{F}\left(\mathcal{E}_{\tilde{W}}\right)-n_{F}\left(\mathcal{E}_{\tilde{H}}^{*}\right)}{\left(\mathcal{E}_{\tilde{W}}-\mathcal{E}_{\tilde{H}}^{*}\right)^{2}}\right. \\
& \left.+\frac{1-n_{F}\left(\mathcal{E}_{\tilde{W}}\right)-n_{F}\left(\mathcal{E}_{\tilde{H}}\right)}{\left(\mathcal{E}_{\tilde{W}}+\mathcal{E}_{\tilde{H}}\right)^{2}}\right\} .
\end{aligned}
$$

The corresponding expression for $S_{\tilde{H}^{0}}^{C P}(x)$ can be obtained by making the same replacements as indicated above for 
the $C P$-conserving terms. The correspondence with the results of Ref. [8] can be seen from Eq. (B7) of Appendix B. We again find essential agreement, apart from a sign difference on the $\cos 2 \phi$ term.

\section{Chiral fermions}

The final source term associated with Fig. 1(a) involves $L$ and $R$ top quarks. At this order, the latter only contribute a $\mu_{i}$-dependent $C P$-conserving term. In order to illustrate the structure of this term that arises when the terms of $\mathcal{O}\left(g^{2}\right)$ are retained, we employ the interacting fermion propagators of Eqs. (28)-(30). The result is:

$$
S_{t_{R}}^{C P}(x)=\Gamma_{t_{R}}^{+}\left(\mu_{t_{L}}+\mu_{t_{R}}\right)+\Gamma_{t_{R}}^{-}\left(\mu_{t_{L}}-\mu_{t_{R}}\right),
$$

with

$$
\begin{aligned}
\Gamma_{t_{R}}^{ \pm}= & \frac{1}{T} \frac{N_{C} y_{t}^{2} v_{u}(x)^{2}}{\pi^{2}} \int_{0}^{\infty} d k k^{2} \operatorname{Im}\left\{\frac { Z _ { p } ^ { R } ( k ) Z _ { p } ^ { L } ( k ) } { \mathcal { E } _ { p } ^ { R } + \mathcal { E } _ { p } ^ { L } } \left[h_{F}\left(\mathcal{E}_{p}^{L}\right)\right.\right. \\
& \left.\mp h_{F}\left(\mathcal{E}_{p}^{R}\right)\right]+\frac{Z_{p}^{L}(k) Z_{h}^{R}(k)^{*}}{\mathcal{E}_{p}^{L}-\mathcal{E}_{h}^{R *}}\left[h_{F}\left(\mathcal{E}_{p}^{L}\right) \mp h_{F}\left(\mathcal{E}_{h}^{R *}\right)\right] \\
& +(p \leftrightarrow h)\} .
\end{aligned}
$$

Here, the " $p$ " and " $h$ " subscripts indicate contributions from the particle and hole modes, and " $L$ " and " $R$ " refer to left- and right-handed quarks. We have not included in our calculation the effects of $\mu_{t_{L R}}$-dependence of the widths $\Gamma_{p, h}^{L, R}\left(T, \mu_{t_{L, R}}\right)$, which in principle also enter at this order. For an expanded version of Eq. (66), including these effects, see Eq. (B8) in Appendix B.

In the limit of $t_{L} \leftrightarrow t_{R}$ symmetry, $\Gamma_{t_{R}}^{+}$vanishes, and $\Gamma_{t_{R}}^{-}$ simplifies to:

$$
\begin{aligned}
\Gamma_{t_{R}}^{-}= & \frac{1}{T} \frac{N_{C} y_{t}^{2} v_{u}(x)^{2}}{\pi^{2}} \int_{0}^{\infty} d k k^{2} \operatorname{Im}\left\{\frac{Z_{p}(k)^{2}}{\mathcal{E}_{p}} h_{F}\left(\mathcal{E}_{p}\right)\right. \\
& +\frac{Z_{h}(k)^{2}}{\mathcal{E}_{h}} h_{F}\left(\mathcal{E}_{h}\right)+\frac{2 Z_{p}(k) Z_{h}^{*}(k)}{\mathcal{E}_{p}-\mathcal{E}_{h}^{*}}\left[h_{F}\left(\mathcal{E}_{p}\right)\right. \\
& \left.\left.+h_{F}\left(\mathcal{E}_{h}^{*}\right)\right]\right\} .
\end{aligned}
$$

We observe that all contributions to the $C P$-violating source terms and the $\Gamma^{ \pm}$vanish in the limit of zero thermal widths. Since the widths are generically of order $g^{2} T$ (here, $g$ denotes either a gauge or Yukawa coupling), the source terms for the QTEs are generally fourth order in the couplings.

\section{QUANTUM TRANSPORT EQUATIONS AND $\rho_{B}$}

We now discuss diffusion equations for the particle species that significantly contribute to the density of lefthanded doublet fermions $n_{L}(x)$ [cf. Eq. (2)] that acts as the "seed" for baryogenesis. We subsequently relate $\rho_{B}$ to $n_{L}$ and solve explicitly the equations in the case of a simple geometry and profile for the bubble wall describing the phase boundary.

\section{A. Solving the diffusion equations}

Using the source terms computed in Section III, one can arrive at a coupled set of differential equations for the various particle number densities. These equations simplify considerably under the assumptions of approximate chemical equilibrium between SM particles and their superpartners $\left(\mu_{f} \approx \mu_{\tilde{f}}\right.$ with $\mu_{\tilde{V}} \approx 0$ ), as well as between different members of left-handed fermion doublets $\left(\mu_{W^{ \pm}} \approx 0\right)$. In this case, one obtains transport equations for densities associated with different members of a supermultiplet. This approach is the one followed in Ref. [10], and for pedagogical purposes we summarize the development here.

First, we define the appropriate supermultiplet densities:

$$
\begin{aligned}
Q \equiv & n_{t_{L}}+n_{\tilde{t}_{L}}+n_{b_{L}}+n_{\tilde{b}_{L}} \\
T \equiv & n_{t_{R}}+n_{\tilde{t}_{R}} \\
B \equiv & n_{b_{R}}+n_{\tilde{b}_{R}} \\
H \equiv & n_{H_{u}^{+}}+n_{H_{u}^{0}}-n_{H_{d}^{-}}-n_{H_{d}^{0}}+n_{\tilde{H}_{u}^{+}}-n_{\tilde{H}_{d}^{-}} \\
& +n_{\tilde{H}_{u}^{0}}-n_{\tilde{H}_{d}^{0}},
\end{aligned}
$$

where the Higgsino densities arise from the vector charges $n_{\tilde{H}^{+}}=\bar{\Psi}_{\tilde{H}^{+}} \gamma^{0} \Psi_{\tilde{H}^{+}}$and $n_{\tilde{H}^{0}}=\bar{\Psi}_{\tilde{H}^{0}} \gamma^{0} \Psi_{\tilde{H}^{0}}$ associated with the Dirac fields defined in Eq. (52). There are analogous definitions for the first- and second-generation (s)quarks. Although we do not consider them here, one may also define the corresponding axial charge densities. In the case of the Higgsinos, for example, it will involve the sum, rather than the difference, of the $u$ - and $d$-type Higgsino densities. ${ }^{5}$

The diffusion equation for a density $n_{i}$ has the structure:

$$
\partial_{\mu} J_{i}^{\mu}=S_{i}^{C P}+S_{i}^{C P}+S_{i}^{\mathrm{sph}},
$$

where $J_{i}^{\mu}$ is the current associated with the density $n_{i}, S_{i}^{C P}$, and $S_{i}^{C P}$ are the source terms computed above, and $S_{i}^{\mathrm{sph}}$ is the sphaleron transition term. Various derivations of the strong sphaleron term appear in the literature, so we do not reproduce them here. However, we note that the expressions in Refs. $[10,27]$ have erroneously omitted a factor of $1 / N_{C}[28]$.

The $C P$-conserving damping terms $S_{i}^{C P}$ have been given in Eqs. (44), (59), and (65) to linear order in the appropriate chemical potentials. Assuming local thermal equilibrium we relate the number densities to the chemical potentials via:

$$
n_{i}=g_{i} \int \frac{d^{3} k}{(2 \pi)^{3}}\left[N\left(\omega_{k}, \mu_{i}\right)-N\left(\omega_{k},-\mu_{i}\right)\right]
$$

\footnotetext{
${ }^{5}$ This density was considered in Ref. [11], and its overall impact on the baryon asymmetry found to be small.
} 
where $N(\omega, \mu)$ is the appropriate boson or fermion distribution function and $g_{i}$ counts the internal degrees of freedom (spin and color). Dropping terms of $\mathcal{O}\left(\mu_{i}^{3}\right)$, one obtains:

$$
n_{i}=\frac{k_{i}\left(m_{i} / T\right) T^{2}}{6} \mu_{i},
$$

where the factors $k_{i}\left(m_{i} / T\right)$ are exponentially small in the regime $m_{i} / T \gg 1$, and reduce in the massless limit to $k_{i}(0)=1$ for chiral fermions, $k_{i}(0)=2$ for Dirac fermions, and $k_{i}(0)=2$ for complex scalars. In our analysis we keep the full dependence on $m_{i} / T$ :

$$
k_{i}\left(m_{i} / T\right)=k_{i}(0) \frac{c_{F, B}}{\pi^{2}} \int_{m / T}^{\infty} d x x \frac{e^{x}}{\left(e^{x} \pm 1\right)^{2}} \sqrt{x^{2}-m^{2} / T^{2}},
$$

where for fermions (bosons) $c_{F(B)}=6(3)$, and we choose the $+(-)$ sign in the denominator.

Using Eq. (71) in Eqs. (44), (59), and (65), and defining:

$$
\begin{aligned}
\Gamma_{\bar{M}}^{ \pm} & =\frac{6}{T^{2}}\left(\Gamma_{t}^{ \pm}+\Gamma_{\tilde{t}}^{ \pm}\right) \\
\Gamma_{h} & =\frac{6}{T^{2}}\left(\Gamma_{\tilde{H}^{ \pm}}+\Gamma_{\tilde{H}^{0}}\right),
\end{aligned}
$$

the resulting set of coupled transport equations is:

$$
\begin{aligned}
\partial^{\mu} T_{\mu}= & \Gamma_{M}^{+}\left(\frac{T}{k_{T}}+\frac{Q}{k_{Q}}\right)-\Gamma_{M}^{-}\left(\frac{T}{k_{T}}-\frac{Q}{k_{Q}}\right) \\
& -\Gamma_{Y}\left(\frac{T}{k_{T}}-\frac{H}{k_{H}}-\frac{Q}{k_{Q}}\right) \\
& +\Gamma_{s s}\left(\frac{2 Q}{k_{Q}}-\frac{T}{k_{T}}+\frac{9(Q+T)}{k_{B}}\right)+S_{\tilde{t}}^{C P} \\
\partial^{\mu} Q_{\mu}= & -\Gamma_{M}^{+}\left(\frac{T}{k_{T}}+\frac{Q}{k_{Q}}\right)+\Gamma_{M}^{-}\left(\frac{T}{k_{T}}-\frac{Q}{k_{Q}}\right) \\
& +\Gamma_{Y}\left(\frac{T}{k_{T}}-\frac{H}{k_{H}}-\frac{Q}{k_{Q}}\right) \\
& -2 \Gamma_{s s}\left(\frac{2 Q}{k_{Q}}-\frac{T}{k_{T}}+\frac{9(Q+T)}{k_{B}}\right)-S_{\tilde{t}}^{C P} \\
\partial^{\mu} H_{\mu}= & -\Gamma_{h} \frac{H}{k_{H}}-\Gamma_{Y}\left(\frac{Q}{k_{Q}}+\frac{H}{k_{H}}-\frac{T}{k_{T}}\right)+S_{\tilde{H}}^{C P},
\end{aligned}
$$

where $\Gamma_{s s}=6 \kappa^{\prime} \frac{8}{3} \alpha_{s}^{4} T$, with $\kappa^{\prime} \sim O(1)$.

In writing down Eqs. (74a)-(74c), we have also included the $H \tilde{q}_{L} \tilde{q}_{R}$ and $H q_{L} q_{R}$ Yukawa interaction term that arises from Fig. 2, though we have not computed the corresponding transport coefficient $\Gamma_{Y}$. In this work we will again follow the authors of Ref. [10], who estimate $\Gamma_{Y} \gg \Gamma_{M}^{-}$. For $\kappa^{\prime} \sim \mathcal{O}(1)$, one also has $\Gamma_{s s} \gg \Gamma_{M}^{ \pm}$. These facts allow one to relate algebraically the densities $Q$ and $T$ to $H$, by setting the linear combinations multiplying $\Gamma_{Y}$ and $\Gamma_{s s}$ equal to $\delta_{Y}=O\left(1 / \Gamma_{Y}\right)$ and $\delta_{s s}=O\left(1 / \Gamma_{s s}\right)$, respectively. One then obtains:

$$
\begin{gathered}
Q=\frac{\left(k_{B}-9 k_{T}\right) k_{Q}}{\left(9 k_{T}+9 k_{Q}+k_{B}\right) k_{H}} H+\alpha_{Q Y} \delta_{Y}+\alpha_{Q s} \delta_{s s} \\
T=\frac{\left(9 k_{T}+2 k_{B}\right) k_{T}}{\left(9 k_{T}+9 k_{Q}+k_{B}\right) k_{H}} H+\alpha_{T Y} \delta_{Y}+\alpha_{T s} \delta_{s s},
\end{gathered}
$$

with known coefficients $\alpha_{Q Y, Q s, T Y, T s}$. Taking $2 \times$ [Eq. (74a)] + [Eq. (74b)] + [Eq. (74c)], introducing the diffusion approximations $\mathbf{T}=-D_{q} \nabla T, \mathbf{Q}=-D_{q} \nabla Q$, $\mathbf{H}=-D_{h} \nabla H$, and using Eq. (75) leads to:

$$
\dot{H}-\bar{D} \nabla^{2} H+\bar{\Gamma} H-\bar{S}=\mathcal{O}\left(\delta_{s s}, \delta_{Y}\right),
$$

where $^{6}$

$\bar{D}=\frac{\left(9 k_{Q} k_{T}+k_{B} k_{Q}+4 k_{T} k_{B}\right) D_{q}+k_{H}\left(9 k_{T}+9 k_{Q}+k_{B}\right) D_{h}}{9 k_{Q} k_{T}+k_{B} k_{Q}+4 k_{T} k_{B}+k_{H}\left(9 k_{T}+9 k_{Q}+k_{B}\right)}$

$$
\bar{\Gamma}=\frac{\left(9 k_{Q}+9 k_{T}+k_{B}\right)\left(\Gamma_{M}^{-}+\Gamma_{h}\right)-\left(3 k_{B}+9 k_{Q}-9 k_{T}\right) \Gamma_{M}^{+}}{9 k_{Q} k_{T}+k_{B} k_{Q}+4 k_{T} k_{B}+k_{H}\left(9 k_{T}+9 k_{Q}+k_{B}\right)}
$$

$$
\begin{aligned}
\bar{S}= & \frac{k_{H}\left(9 k_{Q}+9 k_{T}+k_{B}\right)}{9 k_{Q} k_{T}+k_{B} k_{Q}+4 k_{T} k_{B}+k_{H}\left(9 k_{T}+9 k_{Q}+k_{B}\right)} \\
& \times\left(S_{\tilde{t}}^{C P}+S_{\tilde{H}}^{C P}\right) .
\end{aligned}
$$

The subleading terms $\delta_{Y, s s}$ can be determined by use of Eqs. (75) in Eqs. (74a) and (74b). We include the effect of $\delta_{s s}$ in our final expression for $\rho_{B}$ [10], although its effect is negligible in the relevant MSSM parameter region.

Equation (76) can now be solved for a given set of assumptions about the geometry of the bubble wall. Again, for clarity of illustration, we will work in a framework that allows us to carry analytic calculations as far as possible, leaving to the future a numerical solution of the equations for a realistic wall geometry and profile. First, as commonly done in earlier studies, we ignore the wall curvature, thereby reducing the problem to a onedimensional one in which all relevant functions depend on the variable $\bar{z}=\left|\mathbf{x}+\mathbf{v}_{w} t\right|$, where $\mathbf{v}_{w}$ is the wall velocity. Thus, $\bar{z}<0$ is associated with the unbroken phase, $\bar{z}>0$ with the broken phase, and the boundary wall extends over $0<\bar{z}<L_{w}$. Second, we take the relaxation term $\bar{\Gamma}$ to be nonzero and constant for $\bar{z}>0$. The resulting solution for $H$ in the unbroken phase $\bar{z}<0$ (related to $\rho_{B}$ as shown below) is:

$$
H(\bar{z})=\mathcal{A} e^{v_{w} \bar{z} / \bar{D}}
$$

with

\footnotetext{
${ }^{6}$ Our expressions differ from those in Ref. [10], which we believe result from an algebraic error. The numerical impact of this difference, however, is not significant.
} 


$$
\begin{gathered}
\mathcal{A}=\frac{1}{\bar{D} \kappa_{+}} \int_{0}^{\infty} \bar{S}(y) e^{-\kappa_{+} y} d y, \\
\kappa_{+}=\frac{v_{w}+\sqrt{v_{w}^{2}+4 \bar{\Gamma} \bar{D}}}{2 \bar{D}} \simeq \sqrt{\frac{\bar{\Gamma}}{\bar{D}}} .
\end{gathered}
$$

The above equation is valid for any shape of the source $\bar{S}(\bar{z})$. For simplicity, however, we assume a simple stepfunction type behavior for the source: $\bar{S}$ nonzero and constant for $0<\bar{z}<L_{w}$. Specializing to this case of constant sources in $0<\bar{z}<L_{w}$, using $4 \bar{D} \bar{\Gamma} \gg v_{w}^{2}$, $L_{w} \sqrt{\bar{\Gamma} / \bar{D}} \ll 1$, and taking $\bar{\Gamma}=r_{\Gamma}\left(\Gamma_{h}+\Gamma_{M}^{-}\right)$from Eq. (77a), we arrive at:

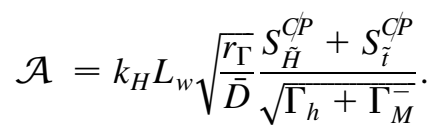

When evaluating the source terms $S_{\tilde{H}}^{Q P}, S_{\tilde{t}}^{C P}$ [see Eqs. (48) and (64)] for this simple profile one has to use $\dot{\beta}=$ $v_{w} \Delta \beta / L_{w}$ : thus $\mathcal{A}$ is explicitly proportional to $v_{w}$ and is only weakly dependent on $L_{w}$. Solutions for $Q$ and $T$ are then obtained via Eqs. (78) and (75).

\section{B. The baryon density $\rho_{B}$}

Neglecting the wall curvature and assuming a stepfunction profile for the weak sphaleron rate, the baryon density satisfies the equation $[12,29]$ :

$$
D_{q} \rho_{B}^{\prime \prime}(\bar{z})-v_{w} \rho_{B}^{\prime}(\bar{z})-\theta(-\bar{z}) \mathcal{R} \rho_{B}=\theta(-\bar{z}) \frac{n_{F}}{2} \Gamma_{\mathrm{ws}} n_{L}(\bar{z}),
$$

where $n_{F}$ is the number of fermion families and the relaxation term is given by [29]:

$$
\mathcal{R}=\Gamma_{\mathrm{ws}}\left[\frac{9}{4}\left(1+\frac{n_{\mathrm{sq}}}{6}\right)^{-1}+\frac{3}{2}\right],
$$

where $n_{\mathrm{sq}}$ indicates the number of light squark flavors, and the weak sphaleron rate is given by $\Gamma_{\mathrm{ws}}=6 \kappa \alpha_{w}^{5} T$, with $\kappa \simeq 20$ [30].

The solution to Eq. (81) in the broken phase, eventually growing into the Universe, is constant and given by:

$$
\rho_{B}=-\frac{n_{F} \Gamma_{\mathrm{ws}}}{2 v_{w}} \int_{-\infty}^{0} n_{L}(x) e^{x \mathcal{R} / v_{w}} d x .
$$

Neglecting leptonic contributions, $n_{L}$ is given in the unbroken phase by the sum of left-handed quark densities over the three generations $\left(Q_{1 L}, Q_{2 L}, Q\right)$. Since appreciable densities of first and second generation quarks are only generated via strong sphaleron processes, it is possible to express $Q_{1 L}$ and $Q_{2 L}$ in terms of $Q$ and $T$, in such a way that $n_{L}=Q+Q_{1 L}+Q_{2 L}=5 Q+4 T$ [10]. Using then Eq. (75) one obtains:

$$
n_{L}=-H\left[r_{1}+r_{2} \frac{v_{w}^{2}}{\Gamma_{\mathrm{ss}} \bar{D}}\left(1-\frac{D_{q}}{\bar{D}}\right)\right]
$$

where

$$
\begin{aligned}
& r_{1}=\frac{9 k_{Q} k_{T}-5 k_{Q} k_{B}-8 k_{T} k_{B}}{k_{H}\left(9 k_{Q}+9 k_{T}+k_{B}\right)} \\
& r_{2}=\frac{k_{B}^{2}\left(5 k_{Q}+4 k_{T}\right)\left(k_{Q}+2 k_{T}\right)}{k_{H}\left(9 k_{Q}+9 k_{T}+k_{B}\right)^{2}},
\end{aligned}
$$

and finally, in the broken phase:

$$
\begin{aligned}
\rho_{B}(\bar{z}>0)= & \frac{n_{F}}{2} \mathcal{A}\left[r_{1} \Gamma_{\mathrm{ws}}+r_{2} \frac{\Gamma_{\mathrm{ws}}}{\Gamma_{\mathrm{ss}}} \frac{v_{w}^{2}}{\bar{D}}\left(1-\frac{D_{q}}{\bar{D}}\right)\right] \\
& \times \frac{2 \bar{D}}{v_{w}\left[v_{w}+\sqrt{\left.v_{w}^{2}+4 \bar{R} \overline{D_{q}}\right]+2 \mathcal{R} \bar{D}}\right.} \\
= & \frac{n_{F}}{2} \mathcal{A}\left[r_{1} \Gamma_{\mathrm{ws}}+r_{2} \frac{\Gamma_{\mathrm{ws}}}{\Gamma_{\mathrm{ss}}} \frac{v_{w}^{2}}{\bar{D}}\left(1-\frac{D_{q}}{\bar{D}}\right)\right] \\
& \times \frac{\bar{D}}{v_{w}^{2}+\mathcal{R}\left(\bar{D}+D_{q}\right)},
\end{aligned}
$$

where the second equality is true in the limit $v_{w}^{2} \gg 4 D_{q} \mathcal{R}$, which holds for the parameters we have chosen in this calculation. The contribution from the first term in Eq. (86) is linear in $v_{w}$, due to the linear dependence on $v_{w}$ contained in the $\dot{\beta}$ appearing in the $C P$-violating sources. The second term is suppressed by two additional powers of $v_{w}$ and generally leads to a negligible contribution to $\rho_{B}$ in the MSSM case (see discussion below). It could, however, be dominant in the case of heavy degenerate $\tilde{t}_{L}$ and $\tilde{t}_{R}$, which leads to $r_{1} \sim 0$ [10].

The central feature emerging from the above discussion is that the net baryon density is proportional to $\mathcal{A} \sim$ $S^{C P} / \sqrt{\Gamma}$. A large relaxation rate $\Gamma$ for the relevant charges will suppress the overall baryon asymmetry. While in Refs. $[8,26]$ it was pointed out how a nonequilibrium quantum transport could result in a resonant enhancement of $S^{C P}$, we observe here that similar resonance effects in the relaxation terms will mitigate the impact of the enhanced sources. In the next section we discuss the numerical impact within the MSSM, but caution that reaching definitive conclusions will require computing the other transport coefficients, such as $\Gamma_{Y}$, within the same framework.

\section{BARYOGENESIS AND ELECTROWEAK PHENOMENOLOGY WITHIN THE MSSM}

The results derived in the previous Sections allow us to perform an illustrative, preliminary analysis of baryogenesis within the MSSM. This should be taken as an exploration, whose robustness will be tested once we implement the next steps in our treatment of the source terms in the transport equations. With this caveat in mind, we explore 
the connections between electroweak baryogenesis and phenomenology within the MSSM, focusing, in particular, on the implications for EDM searches. Throughout, we assume - as in mSUGRA - that all the terms in the Higgs scalar potential and all gaugino masses are real, while all the $A$-parameters (trilinear scalar couplings) are equal at the GUT scale, therefore sharing the same phase $\phi_{A}$. In this case, the baryon asymmetry and EDMs are sensitive to the two independent $C P$-violating phases $\phi_{\mu}$ and $\phi_{A}{ }^{7}$

From the structure of Eqs. (86) and (80) and (48) and (64) we can write the baryon-to-entropy density ratio ${ }^{8}$ $Y_{B} \equiv \rho_{B} / s$ as:

$$
Y_{B}=F_{1} \sin \phi_{\mu}+F_{2} \sin \left(\phi_{\mu}+\phi_{A}\right),
$$

where we have isolated the dependence on the phases $\phi_{\mu}$ and $\phi_{A}$. The first term that contains $F_{1}$ stems from the Higgsino source, while the $F_{2}$ term arises from the squark source.

The functions $F_{1}$ and $F_{2}$ display a common overall dependence on bubble wall parameters $\left(v_{w}, L_{w}, \Delta \beta\right)$, while having distinct dependence on other MSSM mass parameters such as $|\mu|$, the soft mass parameters for gauginos $\left(M_{1,2}\right)$ and squarks $\left(M_{\tilde{t}_{L}}, M_{\tilde{t}_{R}}\right)$, the triscalar coupling $\left|A_{t}\right|$, and $\tan \beta$. In order to assess the size of $Y_{B}$ and the impact on $C P$-violating phases, we must choose a reference region in the MSSM parameter space, and we follow two obvious guidelines: (i) we require that $v\left(T_{c}\right) / T_{c} \gtrsim 1$, so that the baryon asymmetry is not washed out in the broken symmetry phase; (ii) we require no conflict with precision electroweak physics and direct collider searches. Both criteria lead to nontrivial restrictions.

The condition of a strongly first-order phase transition $\left[v\left(T_{c}\right) / T_{c} \geqslant 1\right]$ requires light scalar degrees of freedom coupling to the Higgs sector. It has been shown $[31,32]$ that within the MSSM the only viable candidate is a light top squark, which should be mainly right-handed $\left(\tilde{t}_{R}\right)$ in order to avoid large contributions to the $\rho$ parameter. Quantitatively, for lightest Higgs boson mass $m_{h} \lesssim$ $120 \mathrm{GeV}$, one needs $100 \mathrm{GeV} \lesssim m_{\tilde{t}}<m_{t}$, and sufficiently small stop mixing parameter $\left|A_{t}-\mu / \tan \beta\right| \lesssim 0.6 M_{\tilde{t}_{L}}$ [31]. Moreover, present experimental limits on $m_{h}$ and the constraint $v\left(T_{c}\right) / T_{c} \gtrsim 1$ jointly require either values of $\tan \beta>5$ or $M_{\tilde{t}_{L}}$ in the multi-TeV region [11]. Based on these considerations, for illustrative purposes we work with the following values of MSSM parameters at the electroweak scale: $M_{\tilde{t}_{R}}=0, M_{\tilde{t}_{L}}=1 \mathrm{TeV},\left|A_{t}\right|=200 \mathrm{GeV}$, $M_{2}=200 \mathrm{GeV}, \tan \beta=10$. We also take for the $C P$-odd Higgs mass $m_{A}=150 \mathrm{GeV}$, which translates into $\Delta \beta \sim$

\footnotetext{
${ }^{7}$ One may, of course, work with a more general soft SUSYbreaking sector that contains additional $C P$-violating phases.

${ }^{8} \mathrm{We}$ evaluate the entropy density at the electroweak phase transitions via $s=\left(2 \pi^{2}\right) / 45 \times g_{\text {eff }}(T) T^{3}$, with $g_{\text {eff }}=130.75$, resulting in $s=57.35 T^{3}$. Similarly, to convert the present ratio $\rho_{B} / n_{\gamma}$ to $Y_{B}$, we use the relation $s=7.04 n_{\gamma}$.
}

0.015 [33]. We vary in the plots the scale $|\mu|$, in order to display the resonant behavior for $|\mu| \sim M_{2}$. Finally, for the bubble wall parameters we adopt the central values $v_{w}=$ 0.05 and $L_{w}=25 / T$ [33].

With the above choice of MSSM parameters, the stopinduced contribution to $Y_{B}$ is suppressed $\left(F_{2} \sim 10^{-3} F_{1}\right)$, since one is far off the squark resonance $\left[\left(M_{\tilde{t}_{L}}-M_{\tilde{t}_{R}}\right) \gg\right.$ $\left.M_{\tilde{t}_{R}}\right]$. On the other hand, the Higgsino-induced contribution $F_{1}$ can account for the observed $Y_{B}$ even without maximal values of $\left|\sin \phi_{\mu}\right|$. We highlight below the salient results of our study:

(i) The primary result of our analysis is that both the source $S_{\tilde{H}}^{C P}$ and the relaxation term $\Gamma_{h}$ display the resonant behavior $[8,26]$ typical of quantum transport for $|\mu| \sim M_{2}$. We illustrate this in Fig. 4: the left panel shows the behavior of the rescaled $C P$-violating Higgsino source $\hat{S}_{\tilde{H}} \equiv$ $-S_{\tilde{H}}^{C P} /\left(v^{2} \dot{\beta} \sin \phi_{\mu}\right)$ versus $|\mu|$, while the right panel displays the ratio $R_{\Gamma}$ of the relaxation term $\left(\Gamma_{h}+\Gamma_{M}^{-}\right)$as calculated in this work to the one used in previous studies, $\left(\Gamma_{h}+\Gamma_{M}^{-}\right)_{\text {H.N. [10]. To our }}$ knowledge this is the first explicit calculation showing resonance behavior for the relaxation term $\bar{\Gamma} \sim r_{\Gamma}\left(\Gamma_{h}+\Gamma_{M}^{-}\right)$.

(ii) Since $F_{1}$ is proportional to $S_{\tilde{H}}^{C P} / \sqrt{\Gamma_{h}+\Gamma_{M}^{-}}$, the baryon asymmetry retains a resonant behavior, albeit with an attenuation of the peak due to the enhanced relaxation term. This is shown explicitly in Fig. 5. In the left panel we plot $F_{1} / Y_{B}^{\text {WMAP }}$, normalizing to the baryon asymmetry extracted from CMB studies [3]: $Y_{B}^{\mathrm{WMAP}}=(9.2 \pm 1.1) \times$ $10^{-11}$ (the quoted error corresponds to $95 \% \mathrm{CL}$ ).

(iii) For completeness we also display in Fig. 5 (right panel) the behavior of the squark contribution $F_{2} / Y_{B}^{\text {WMAP }}$ as a function of $M_{\tilde{t}_{L}}$, with $M_{\tilde{t}_{R}}=$ $100 \mathrm{GeV}$. Within the MSSM, precision electroweak data and the requirement that $v\left(T_{c}\right) / T_{c} \gtrsim 1$ force the masses to be far away from the peak region. However, in extensions of the MSSM where the phase transition is strengthened by additional scalar degrees of freedom this contribution might be important (see, e.g., Refs. [4,5]).

(iv) For given values of the MSSM parameter space explored here, successful EWB carves out a band in $\left|\sin \phi_{\mu}\right|$ centered at $\left|\sin \phi_{\mu}\right|=Y_{B}^{\exp } /\left|F_{1}\right|$ (whose width depends on the uncertainty in $Y_{B}^{\exp }$ ). Because of the resonant behavior of $F_{1}$, the location of this band is highly sensitive to the relative size of $M_{2}$ and $|\mu|$. As illustration, in Fig. 6 we plot the allowed band in the $\left|\sin \phi_{\mu}\right|-|\mu|$ plane determined by the baryon asymmetry, with all other MSSM parameters fixed as above. The bands in the plot combined together correspond to the baryon density determined from Big Bang Nucleosynthesis, $Y_{B}^{\mathrm{BBN}}=(7.3 \pm 2.5) \times 10^{-11}$ (the 


\section{$\hat{S}_{\tilde{H}}$}

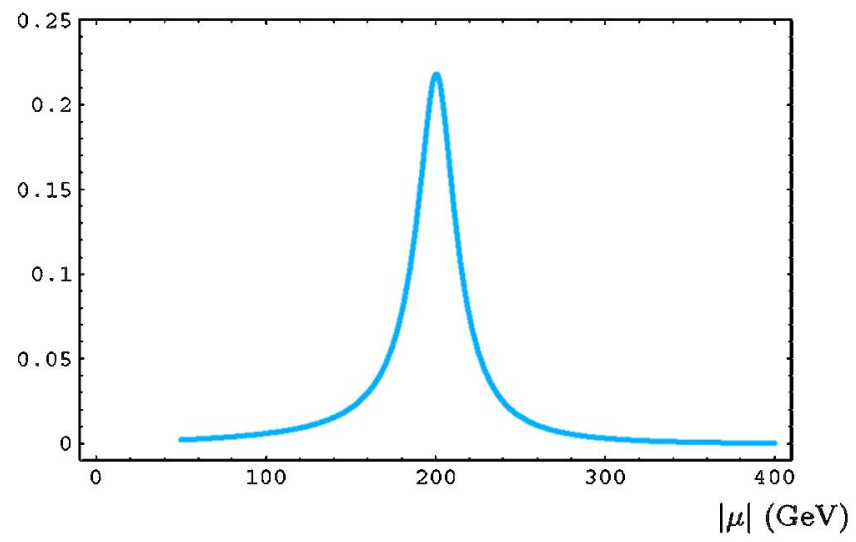

$R_{\Gamma}$

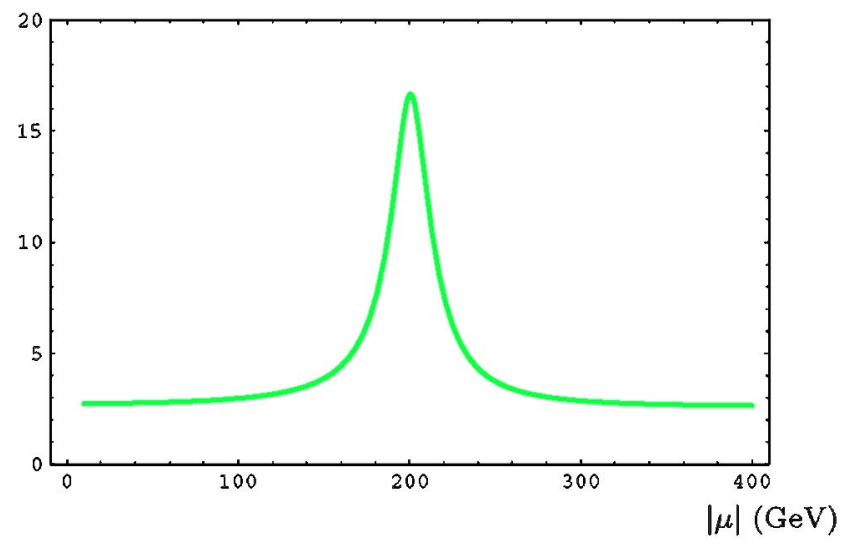

FIG. 4 (color online). Left panel: $C P$-violating Higgsino source $\hat{S}_{\tilde{H}}=-S_{\tilde{H}}^{C P} /\left(v^{2} \dot{\beta} \sin \phi_{\mu}\right) \cos$, as a function of $|\mu|$. Right panel: relaxation rate $R_{\Gamma}=\left(\Gamma_{h}+\Gamma_{M}^{-}\right) /\left(\Gamma_{h}+\Gamma_{m}\right)_{\text {H.N. }}$, normalized to the value used in [10], as a function of $|\mu|$. We have taken $M_{2}=$ $200 \mathrm{GeV}$, and the values of all other parameters as indicated in the text.
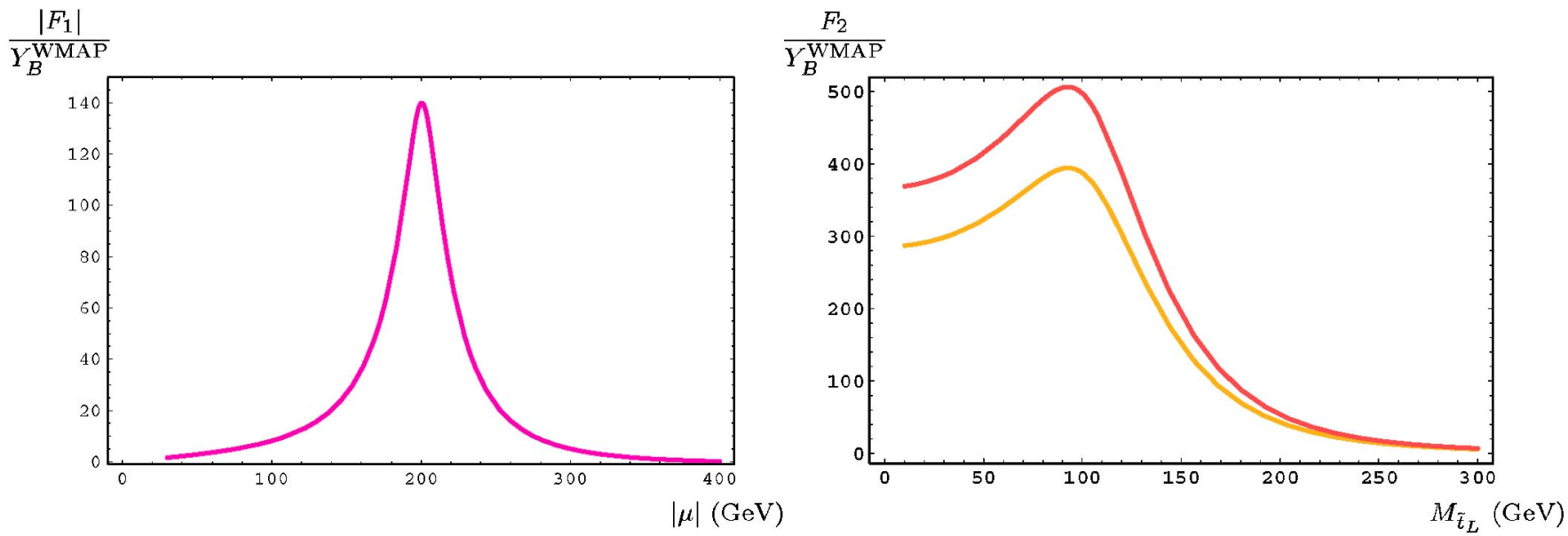

FIG. 5 (color online). Left panel: Higgsino contribution to $Y_{B}$ (Cf. Eq. (87)), normalized to the observed value. $F_{1}$ displays residual resonant behavior for $|\mu| \sim M_{2}$. All other input parameters are given in the text. Right panel: Stop contribution to $Y_{B}$ (Cf. Eq. (87)) normalized to the observed value. The upper curve is for $M_{\tilde{b}_{L}}=M_{\tilde{t}_{L}}$, while the lower one is for $M_{\tilde{b}_{L}} \gg M_{\tilde{t}_{L}}$. We have taken here $M_{\tilde{t}_{R}}=100 \mathrm{GeV},|\mu|=200 \mathrm{GeV}$, and have allowed $M_{\tilde{t}_{L}}$ to reach unrealistically low values to explore the size of the squark resonance. For realistic input parameters $F_{2} \ll F_{1}$.

error corresponds to $95 \%$ CL [2]). Using WMAP input leads to the narrow, lighter-shaded band in our plot located at the upper edge of the BBNinduced band.

We conclude this section with a brief account of the connections between the baryon asymmetry and EDM phenomenology. Since the Standard Model predictions are in general highly suppressed and well below present experimental sensitivity, limits on the electron, neutron, and atomic EDMs can be used to constrain the phases of a given new physics model. Present limits of interest to us are those for the electron [34] and ${ }^{199} \mathrm{Hg}$ [35] EDMs:

$$
\begin{aligned}
\left|d_{e}\right| & <1.9 \times 10^{-27} e \cdot \mathrm{cm}(95 \% \mathrm{CL}), \\
\left|d_{\mathrm{Hg}}\right| & <2.1 \times 10^{-28} e \cdot \mathrm{cm}(95 \% \mathrm{CL}) .
\end{aligned}
$$

Although a single EDM can be sufficiently small even for maximally large $C P$-violating phases (due to cancellations), constraints from more than one EDM can be very powerful. In Ref. [36], for example, it was pointed out how limits on electron and ${ }^{199} \mathrm{Hg}$ EDMs single out a well defined region in the $\phi_{\mu}-\phi_{A}$ plane, for given values of gauginos, squark and slepton masses. As shown above, for each point in the MSSM parameter space, electroweak baryogenesis also selects a band in the $\phi_{\mu}-\phi_{A}$ plane. 


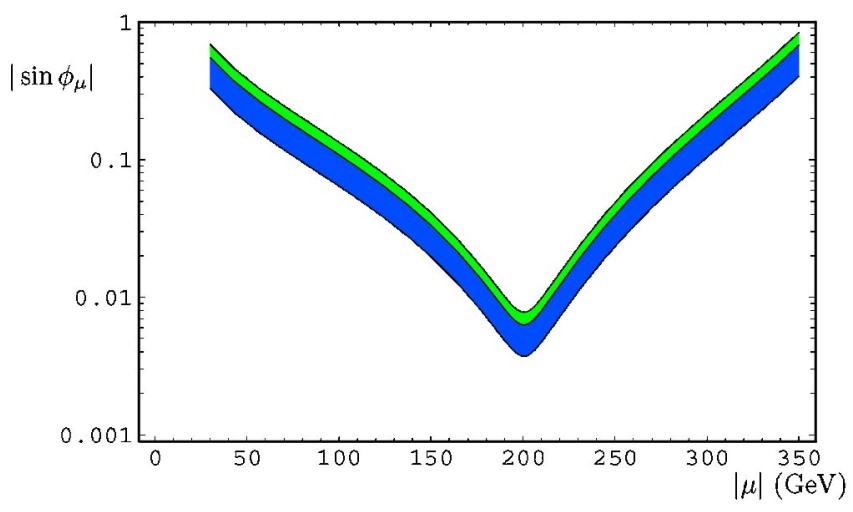

FIG. 6 (color online). Allowed band in the $\left|\sin \phi_{\mu}\right|-|\mu|$ plane, obtained by requiring successful electroweak baryogenesis. All other MSSM parameters are given in the text. The light-shaded narrow band corresponds to the experimental input from WMAP, while the two bands combined [dark + light] correspond to input from Big Bang Nucleosynthesis.

This implies in general nontrivial constraints on the MSSM parameter space, as the EDM-allowed region need not in general coincide with the one required by the baryon asymmetry.

To illustrate this situation, we have evaluated the bands in $\phi_{\mu}-\phi_{A}$ allowed by present limits on electron EDM, mercury EDM, and EWB for two representative points in the MSSM parameter space (see Fig. 7). In our analysis we take the expressions for the electron EDM and quark chromo-electric dipole moments from Ref. [37]. In relating the ${ }^{199} \mathrm{Hg}$ EDM to the quark-level $C P$-violating couplings, we follow the treatment of Ref. [36], ${ }^{9}$ where it was shown that the dominant contribution arises from the chromoelectric dipole moments of quarks $\left(\tilde{d}_{q}\right)$ according to

$$
d_{\mathrm{Hg}}=-\left(\tilde{d}_{d}-\tilde{d}_{u}-0.012 \tilde{d}_{s}\right) \times 3.2 \cdot 10^{-2} e .
$$

According to the same authors, the analysis of the neutron EDM involves additional effects, such as the $C P$-violating three-gluon operator $\tilde{G} G G$, that require a detailed analysis going beyond the scope of the present work. Although the experimental bounds on the neutron EDM - as well as the prospects for future improvements - are competitive with those for the electron and neutral atoms, we defer an analysis of its implications for EWB to a future study. We also neglect the renormalization group evolution of $\phi_{\mu}$ and $\phi_{A}$ from the weak scale to the atomic scale, having assumed a common, flavor-independent phase for the triscalar coupling at the former.

The plots in Fig. 7 correspond to taking the first and second generation sfermions, along with the gluinos, to be degenerate with masses equal to $750 \mathrm{GeV}$; the gaugino mass $M_{1}=100 \mathrm{GeV}$; and the triscalar coupling

\footnotetext{
${ }^{9}$ For a recent reanalysis of hadronic EDMs in SUSY see Ref. [38].
}

$A=200 \mathrm{GeV}$. We consider then two cases for $M_{2}$ and $\mu$ : the left panel corresponds to the resonance peak $M_{2}=|\mu|=200 \mathrm{GeV}$, while the right panel corresponds to off-resonance parameters $M_{2}=200 \mathrm{GeV}$, $|\mu|=250 \mathrm{GeV}$. For these choices of MSSM parameters, Eq. (87) predicts for $Y_{B}$ :

$$
\begin{aligned}
M_{2} & =\mu=200 \mathrm{GeV}: \\
Y_{B} & =-1.3 \times 10^{-8} \sin \phi_{\mu}+1.7 \times 10^{-11} \sin \left(\phi_{A}+\phi_{\mu}\right), \\
M_{2} & =200 \mathrm{GeV}, \quad \mu=250 \mathrm{GeV}: \\
Y_{B} & =-2.0 \times 10^{-9} \sin \phi_{\mu}+4.6 \times 10^{-11} \sin \left(\phi_{A}+\phi_{\mu}\right) .
\end{aligned}
$$

These cases illustrate the main trend: for $M_{2} \sim|\mu|$ electroweak baryogenesis requires relatively small phases, and is consistent with the constraints from EDMs. As one moves off resonance, then larger phases are needed to generate the observed baryon asymmetry, and this requirement tends to conflict with the EDM constraints. Indeed, within our simplified analysis, we find that baryogenesis and EDM constraints become inconsistent for $|\mu|-M_{2} \gtrsim$ $50 \mathrm{GeV}$, when all other superpartners are kept around $750 \mathrm{GeV}$. Of course, increasing (decreasing) the sfermion masses relaxes (tightens) the EDM limits on $C P$-violating phases and affects the above conclusion.

Ultimately, if supersymmetry is discovered at collider experiments, spectroscopy will dictate the input for mass parameters. Then joint constraints from low-energy EDM measurements and collider searches could be used to tightly test the scenario of baryogenesis at the electroweak scale. In particular, we note that a new generation of lepton, neutron, and neutral atom EDM searches will likely tighten the constraints in Fig. 7 by two or more orders of magnitude (for a recent discussion, see Ref. [39] and references therein).

\section{CONCLUSIONS}

It is instructive to consider the essential physics leading to the enhanced sources and relaxation terms discussed in this work. The propagation of quasiparticles in the plasma is modified by scattering from the spacetime varying Higgs vevs that causes transitions to intermediate states involving other quasiparticle species. The system retains some memory of each scattering due to the presence of thermal widths, $\Gamma_{i}$, that reflect the degeneracy of states in the thermal bath. For $\Gamma_{i}=0$, the oscillating exponentials appearing in the Green's functions wash out any memory of the scattering. For $\Gamma_{i} \neq 0$, the Green's functions now contain decaying exponentials as well as oscillating terms, and the memory washout is incomplete. The impact of quantum memory effects are, thus, characterized by the ratio of time scales, $\tau_{\text {int }} / \tau_{p} \sim \Gamma_{i} / \omega_{i}$, where $\tau_{\text {int }}$ is the characteristic propagation time associated with a quasiparticle of frequency $\omega_{i}$ and $\tau_{p} \sim 1 / \Gamma_{i}$, the plasma time, is the time scale on which transitions between the 

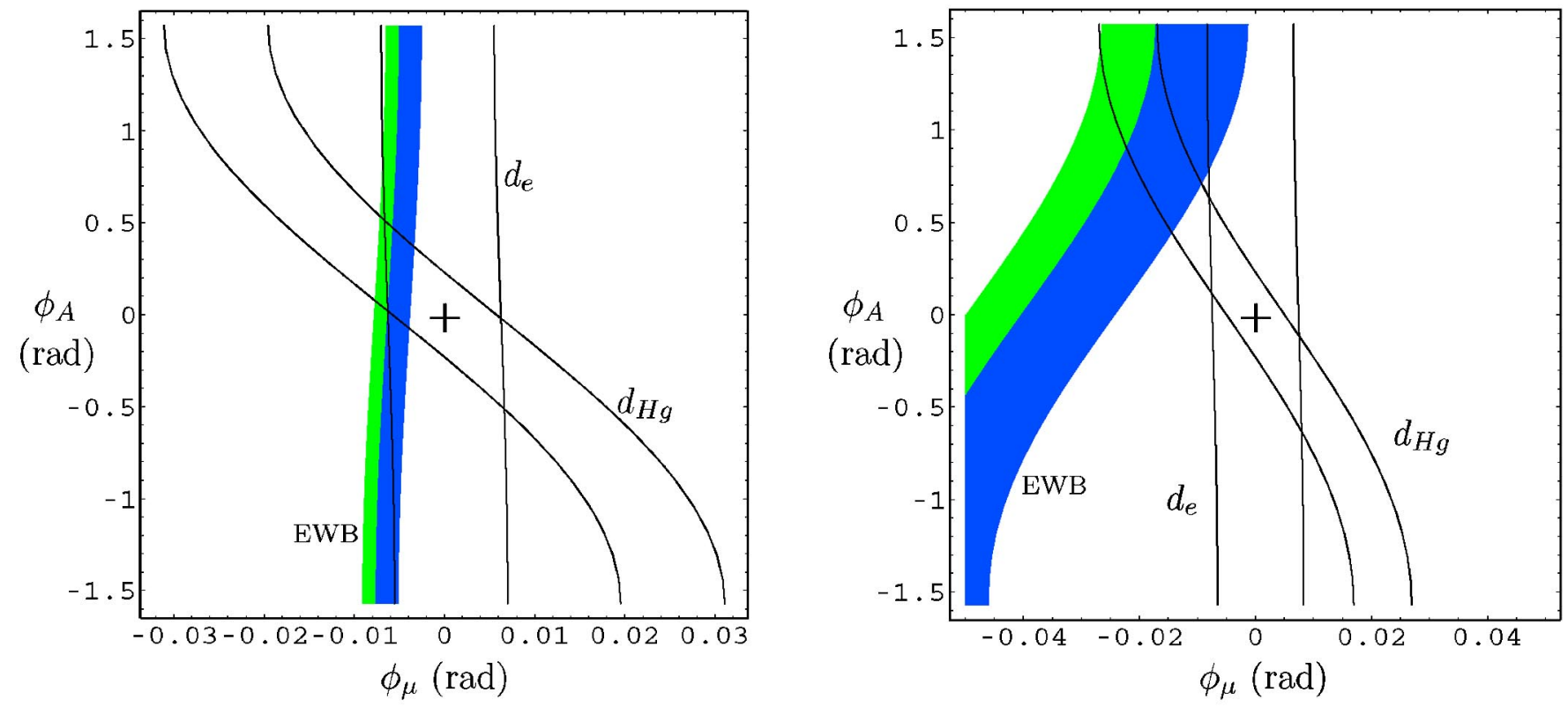

FIG. 7 (color online). Allowed bands in the $\phi_{\mu}-\phi_{A}$ plane implied by consistency with the 95\% C.L. limits on electron EDM $\left(\left|d_{e}\right|<1.9 \times 10^{-27} e \cdot \mathrm{cm}[34]\right)$, mercury $\operatorname{EDM}\left(\left|d_{\mathrm{Hg}}\right|<2.1 \times 10^{-28} e \cdot \mathrm{cm}\right.$ [35]), and baryogenesis. The shaded [dark and light combined] EWB band corresponds to BBN input [2], while the narrow light-shaded band on the left corresponds to WMAP input [3]. In the left panel we use $|\mu|=M_{2}=200 \mathrm{GeV}$ (resonance peak), while in the right panel we use $M_{2}=200 \mathrm{GeV}$ and $|\mu|=250 \mathrm{GeV}$ (off resonance). In both cases the other supersymmetric masses are as specified in the text.

quasiparticle and other, degenerate states may occur. To the extent that the quasiparticle thermal mass and/or three-momentum is large compared to $\Gamma_{i}$, this ratio $\tau_{\text {int }} / \tau_{p}$ is $\mathcal{O}(\epsilon)$.

A special situation arises, however, when the spacetime variation of the Higgs vevs is gentle and the thermal mass of an intermediate state is close to that of the initial state. Under these conditions, the scattering event injects essentially zero four-momentum into the initial state $i$, leading to resonant production of the intermediate state $j$. The characteristic lifetime of the latter is no longer $\tau_{\text {int }} \sim 1 / \omega_{i}$, but rather the resonance time scale

$$
\tau_{\text {res }} \sim \frac{1}{\sqrt{\Delta \omega^{2}+\Gamma_{i j}^{2}}},
$$

where $\Delta \omega=\omega_{i}-\omega_{j}$ and $\Gamma_{i j}=\Gamma_{i}+\Gamma_{j} \quad$ [see, e.g., Eqs. (B1)-(B3) and (B5)-(B7) of Appendix B]. In this case, the impact of quantum memory is characterized by the ratio $\tau_{\text {res }} / \tau_{p}$. For $|\Delta \omega| \ll \Gamma_{i j}$, this ratio becomes of $\mathcal{O}(1)$, and the impact of quantum memory is resonantly enhanced. ${ }^{10}$ On the other hand, for $|\Delta \omega| \gg \Gamma_{i j}$, the ratio is $\mathcal{O}(\epsilon)$ and one returns to the more generic conditions.

\footnotetext{
${ }^{10}$ An examination of Eqs. (B1)-(B3) and (B5)-(B7) of Appendix $\mathrm{B}$ indicates the presence of an additional, dynamical enhancement factor $\sim \omega / \sqrt{\Delta \omega^{2}+\Gamma_{i j}^{2}}$ in the relevant integrals.
}

In this study, we have shown how this effect can enhance both the particle number-changing relaxation terms as well as the $C P$-violating sources that enter the transport equations relevant to electroweak baryogenesis. Importantly, the effect of resonant relaxation tends to mitigate the impact of resonantly enhanced sources, as both enhancements occur under the same conditions for the electroweak model parameters (in this case, those of the MSSM). We suspect that analogous resonant effects occur in other transport coefficients, such as the $\Gamma_{Y}$ Yukawa terms discussed above, but that the conditions on model parameters leading to enhancements - owing to simple kinematic considerations - will be different. It may be, for example, that the Yukawa interactions are no longer fast compared to the Higgs vev induced transitions when the latter are resonantly enhanced, and in this case, the solution to the differential equations will differ from the general structure obtained here and by other authors. This possibility is one that should be explored in future work.

Additional refinements of the present analysis are clearly in order, including some form of all-orders resummation of the Higgs vev insertions (possibly along the lines proposed in Refs. [11,25]) and a treatment of the axial charge transport equations via Eq. (20). In principle, one would also like to study the density dependence of the thermal frequencies and widths, the impact of nonzero gaugino densities, variations in bubble wall geometry, and possibly higher-order effects in $\epsilon$, such as the departure of $\delta f$ of the thermal distribution functions from their 
equilibrium values. In short, it is apparent that EWB is not yet a solved problem, but rather one that calls for additional study.

Undertaking this effort will be important for electroweak phenomenology. As illustrated here as well as in other studies (e.g., [40]), determining the viability of EWB within a given electroweak model involves a detailed interplay of collider phenomenology, precision electroweak data, EDM searches, and a careful treatment of the dynamics of the electroweak phase transition. In particular, in light of the open questions pertaining to the latter, it is too soon to draw definitive conclusions about the implications of the next generation of EDM searches for the baryon asymmetry. One hopes, however, that by the time these searches obtain their first results, the context for their theoretical interpretation will have been further clarified.

\section{ACKNOWLEDGMENTS}

We are grateful to Christian Bauer, Gerald Dunne, and Mark Wise for useful discussions and to Michael Graesser for detailed reading of the manuscript and useful feedback. The authors are grateful for the support from the Institute for Nuclear Theory, where part of this work was completed. This work was supported in part by the U.S. Department of Energy under Contracts No. DE-FG0302ER41215 and No. DE-FG03-92ER40701, in part by the National Science Foundation under Grant No. PHY00-71856, and in part by the U.S. Department of Defense (C.L.).

\section{APPENDIX A: PROPAGATORS AT FINITE TEMPERATURE AND DENSITY}

In this section, we derive some useful properties of propagators at finite temperature and density, using derivations based on those for the case of finite temperature and zero density in Refs. [16,18].

\section{General Structure of Fermion Propagators}

We begin with the spectral function for fermions at temperature $T=1 / \beta$ in the presence of a chemical potential $\mu$ :

$$
\rho_{\alpha \beta}(x)=\frac{1}{Z} \operatorname{Tr}\left[e^{-\beta(H-\mu N)}\left\{\psi_{\alpha}(x), \bar{\psi}_{\beta}(0)\right\}\right],
$$

where $Z=\operatorname{Tr}\left[e^{-\beta(\mathrm{H}-\mu \mathrm{N})}\right]$. It is convenient to define the retarded and advanced propagators:

$$
\begin{aligned}
& S^{R}(x)=\theta\left(x^{0}\right) \rho(x) \\
& S^{A}(x)=-\theta\left(x^{0}\right) \rho(x),
\end{aligned}
$$

suppressing spinor indices. The Fourier transforms of $S^{R, A}(x)$ and $\rho(x)$ are related by:

$$
\begin{aligned}
& S^{R}\left(k^{0}, \mathbf{k}\right)=i \int_{-\infty}^{\infty} \frac{d \omega}{2 \pi} \frac{\rho(\omega, \mathbf{k})}{k^{0}-\omega+i \epsilon} \\
& S^{A}\left(k^{0}, \mathbf{k}\right)=i \int_{-\infty}^{\infty} \frac{d \omega}{2 \pi} \frac{\rho(\omega, \mathbf{k})}{k^{0}-\omega-i \epsilon} .
\end{aligned}
$$

It is possible to express the momentum-space spectral function in terms of a single product of $\psi_{\alpha}(x)$ and $\bar{\psi}_{\beta}(x)$ instead of the anticommutator in Eq. (A1), whose Fourier transform is:

$$
\begin{aligned}
\rho_{\alpha \beta}(\omega, \mathbf{k})= & \int d^{4} x e^{i(\omega t-\mathbf{k} \cdot \mathbf{x})} \rho_{\alpha \beta}(t, \mathbf{x}) \\
= & \int d^{4} x e^{i(\omega t-\mathbf{k} \cdot \mathbf{x})} \frac{1}{Z} \sum_{n}\langle n| e^{-\beta(\mathrm{H}-\mu \mathrm{N})}\left[\psi_{\alpha}(x) \bar{\psi}_{\beta}(0)\right. \\
& \left.+\bar{\psi}_{\beta}(0) \psi_{\alpha}(x)\right]|n\rangle .
\end{aligned}
$$

Now insert a complete set of states between the fermion fields:

$$
\begin{aligned}
\rho(\omega, \mathbf{k})= & \int d^{4} x e^{i(\omega t-\mathbf{k} \cdot \mathbf{x})} \frac{1}{Z} \sum_{n, j}\left[\left\langle n\left|e^{-\beta(\mathrm{H}-\mu \mathrm{N})} \psi_{\alpha}(x)\right| j\right\rangle\right. \\
& \times\left\langle j\left|\bar{\psi}_{\beta}(0)\right| n\right\rangle+\left\langle n\left|e^{-\beta(\mathrm{H}-\mu \mathrm{N})} \bar{\psi}_{\beta}(0)\right| j\right\rangle \\
& \left.\times\left\langle j\left|\psi_{\alpha}(x)\right| n\right\rangle\right] .
\end{aligned}
$$

We can rewrite the second term by switching summation labels and translating $\psi_{\alpha}$ from $x$ to 0 :

$$
\begin{aligned}
\sum_{n, j}\left\langle n\left|e^{-\beta(\mathrm{H}-\mu \mathrm{N})} \bar{\psi}_{\beta}(0)\right| j\right\rangle\left\langle j\left|\psi_{\alpha}(x)\right| n\right\rangle \\
=\sum_{j, n} e^{i\left(E_{n}-E_{j}\right) t} e^{-i\left(\mathbf{k}_{n}-\mathbf{k}_{j}\right) \cdot \mathbf{x}} e^{-\beta E_{j}} e^{\beta \mu\left(N_{n}+1\right)}\left\langle n\left|\psi_{\alpha}(0)\right| j\right\rangle \\
\quad \times\left\langle j\left|\bar{\psi}_{\beta}(0)\right| n\right\rangle,
\end{aligned}
$$

which after integrating in Eq. (A5), becomes

$$
\begin{aligned}
& \frac{1}{Z} \sum_{j, n}(2 \pi)^{4} \delta\left(\omega+E_{n}-E_{j}\right) \delta^{3}\left(\mathbf{k}+\mathbf{k}_{n}-\mathbf{k}_{j}\right) \\
& \quad \times e^{-\beta\left(E_{n}+\omega\right)} e^{\beta \mu\left(N_{n}+1\right)}\left\langle n\left|\psi_{\alpha}(0)\right| j\right\rangle\left\langle j\left|\bar{\psi}_{\beta}(0)\right| n\right\rangle,
\end{aligned}
$$

where we used the first delta function to replace $E_{j}$ with $E_{n}+\omega$ in the exponential $e^{-\beta E_{j}}$. This can now be written:

$$
\begin{aligned}
e^{-\beta(\omega-\mu)} \frac{1}{Z} \int d^{4} x e^{i(\omega t-\mathbf{k} \cdot \mathbf{x})} \sum_{n, j}\left\langle n\left|e^{-\beta(\mathrm{H}-\mu \mathrm{N})} \psi_{\alpha}(x)\right| j\right\rangle \\
\times\left\langle j\left|\bar{\psi}_{\beta}(0)\right| n\right\rangle
\end{aligned}
$$

which is $e^{-\beta(\omega-\mu)}$ times the first term of Eq. (A5), so we conclude:

$$
\begin{aligned}
\rho(\omega, \mathbf{k})= & {\left[1+e^{-\beta(\omega-\mu)}\right] } \\
& \times \int d^{4} x e^{i(\omega t-\mathbf{k} \cdot \mathbf{x})} \frac{1}{Z} \operatorname{Tr}\left[e^{-\beta(\mathrm{H}-\mu \mathrm{N})} \psi_{\alpha}(x) \bar{\psi}_{\beta}(0)\right] .
\end{aligned}
$$

Similarly, we could have manipulated the first term of Eq. (A5) in the same way, and derived the companion relation: 


$$
\begin{aligned}
\rho(\omega, \mathbf{k})= & {\left[1+e^{\beta(\omega-\mu)}\right] } \\
& \times \int d^{4} x e^{i(\omega t-\mathbf{k} \cdot \mathbf{x})} \frac{1}{Z} \operatorname{Tr}\left[e^{-\beta(\mathrm{H}-\mu \mathrm{N})} \bar{\psi}_{\beta}(0) \psi_{\alpha}(x)\right] .
\end{aligned}
$$

The Green's functions $S^{>}\left(k^{0}, \mathbf{k}\right)$ and $-S^{<}\left(k^{0}, \mathbf{k}\right)$ appear on the right-hand sides of Eqs. (A9) and (A10), giving the relations:

$$
\begin{aligned}
& S^{>}\left(k^{0}, \mathbf{k}\right)=\left[1-n_{F}\left(k^{0}-\mu\right)\right] \rho\left(k^{0}, \mathbf{k}\right) \\
& S^{<}\left(k^{0}, \mathbf{k}\right)=-n_{F}\left(k^{0}-\mu\right) \rho\left(k^{0}, \mathbf{k}\right),
\end{aligned}
$$

where $n_{F}(x)=1 /\left(1+e^{x}\right)$.

The various Green's functions satisfy the identities:

$$
S^{t}(x, y)=S^{R}(x, y)+S^{<}(x, y)=S^{A}(x, y)+S^{>}(x, y)
$$

$S^{\bar{t}}(x, y)=S^{>}(x, y)-S^{R}(x, y)=S^{<}(x, y)-S^{A}(x, y)$,

which follow directly from the definitions in Eqs. (8) and (A2). Thus, using Eq. (A11), the time- and anti-timeordered propagators can be expressed in terms of the retarded and advanced propagators:

$$
\begin{aligned}
S^{t}\left(k^{0}, \mathbf{k}\right)= & {\left[1-n_{F}\left(k^{0}-\mu\right)\right] S^{R}\left(k^{0}, \mathbf{k}\right) } \\
& +n_{F}\left(k^{0}-\mu\right) S^{A}\left(k^{0}, \mathbf{k}\right) \\
S^{\bar{t}}\left(k^{0}, \mathbf{k}\right)= & -n_{F}\left(k^{0}-\mu\right) S^{R}\left(k^{0}, \mathbf{k}\right) \\
& -\left[1-n_{F}\left(k^{0}-\mu\right)\right] S^{A}\left(k^{0}, \mathbf{k}\right) .
\end{aligned}
$$

Also note that $\rho=S^{R}-S^{A}=S^{>}-S^{<}$.

\section{Bosonic Propagators}

Similar results may be derived from scalar bosonic propagators, for which the analog to Eq. (A11) is:

$$
\begin{aligned}
& G^{>}\left(k^{0}, \mathbf{k}\right)=\left[1+n_{B}\left(k^{0}-\mu\right)\right] \rho\left(k^{0}, \mathbf{k}\right) \\
& G^{<}\left(k^{0}, \mathbf{k}\right)=n_{B}\left(k^{0}-\mu\right) \rho\left(k^{0}, \mathbf{k}\right),
\end{aligned}
$$

where the momentum-space spectral function $\rho\left(k^{0}, \mathbf{k}\right)$ for bosons is the Fourier transform of:

$$
\rho(x)=\frac{1}{Z} \operatorname{Tr}\left\{e^{-\beta(\mathrm{H}-\mu \mathrm{N})}\left[\phi(x), \phi^{*}(0)\right]\right\} .
$$

The bosonic propagators also satisfy the identity $\rho=$ $G^{R}-G^{A}=G^{>}-G^{<}$.

\section{Tree-Level Propagators}

At tree level, the propagators $S^{R, A}$ for fermions are given by:

$$
S^{R, A}\left(k^{0}, \mathbf{k}\right)=\frac{i(k+m)}{\left(k^{0} \pm i \epsilon\right)^{2}-E_{\mathbf{k}}^{2}}
$$

and $G^{R, A}$ for bosons are given by:

$$
G^{R, A}\left(k^{0}, \mathbf{k}\right)=\frac{i}{\left(k^{0} \pm i \epsilon\right)^{2}-E_{\mathbf{k}}^{2}}
$$

where $E_{\mathbf{k}}^{2}=|\mathbf{k}|^{2}+m^{2}$. Note that these propagators are independent of the temperature and chemical potential, which only enter in the thermal distribution functions appearing in the relations of the retarded and advanced propagators to the other Green's functions, for example, in Eq. (A13).

\section{One-Loop Corrections to Massless Fermion Propagators}

Resumming the one-loop self-energy into the fermion propagator at finite temperature changes the pole structure of the propagator dramatically, introducing a new collective hole excitation of the plasma $[16,17]$. In fact, this structure can be shown to hold even beyond perturbation theory [18]. Extending the results of Ref. [18] to include dependence on a chemical potential, the propagator takes the form given in Eqs. (28)-(30). Recall that in those equations $\mathcal{E}_{p, h}=\omega_{p, h}-i \Gamma_{p, h}$ are the complex poles of the spectral function, and $Z_{p, h}$ are the corresponding residues. At leading order in the "hard thermal loop" approximation (see Ref. [14]), calculating the poles only to order $\mathcal{E} \sim g T$, one finds $\Gamma=0$, and $Z_{p, h}(k, \mu)$ and $\omega_{p, h}(k, \mu)$, where $k=|\mathbf{k}|$, depend only quadratically on $\mu / T$, which we thus neglect in our analysis in the present work, where we keep only effects linear in $\mu / T$. In this limit, and including only a single gluon loop in the quark self-energy diagram, the poles of the spectral function are given by the solutions to the equation:

$$
0=k^{0}-k-\frac{\alpha_{s} C_{F} \pi T^{2}}{4 k}\left[\left(1-\frac{k^{0}}{k}\right) \log \left|\frac{k^{0}+k}{k^{0}-k}\right|+2\right]
$$

where $C_{F}=4 / 3$ is the Casimir of the fundamental representation of $S U(3)$. The solutions to this equation give the poles $k^{0}=E_{p}(k),-E_{h}(k)$. The residues satisfy:

$$
Z_{p, h}(k)=\frac{E_{p, h}^{2}-k^{2}}{m_{f}^{2}}
$$

where

$$
m_{f}^{2}=\frac{\alpha_{s} C_{F} \pi T^{2}}{2}
$$

Calculation of the imaginary parts $\Gamma_{p, h}$ of the poles, since they begin at order $g^{2} T$, requires a resummation of hard thermal loops in self-energy diagrams [19-21]. We are also interested in their dependence on the chemical potential $\mu$. We leave the calculation of these effects to a future study. 
APPENDIX B: EXPANDED SOURCE TERMS FOR QUANTUM TRANSPORT

\section{Bosons}

The $C P$-conserving source term for right-handed stops in Eq. (42) can be expanded by explicitly taking the imaginary part of the integrand:

$$
\begin{aligned}
S_{\tilde{t}_{R}}^{C P}(x)= & -\frac{1}{T} \frac{N_{C} y_{t}^{2}}{2 \pi^{2}}\left|A_{t} v_{u}(x)-\mu^{*} v_{d}(x)\right|^{2} \int_{0}^{\infty} \frac{k^{2} d k}{\omega_{R} \omega_{L}} \\
& \times\left\{\mu _ { R } \left[\frac{1}{\Delta}\left(\sin \phi \operatorname{Im} h_{R}^{+}+\cos \phi \operatorname{Re} h_{R}^{+}\right)\right.\right. \\
& \left.-\frac{1}{\delta}\left(\cos \theta \operatorname{Re} h_{R}^{+}-\sin \theta \operatorname{Im} h_{R}^{+}\right)\right] \\
& +\mu_{L}\left[\frac{1}{\Delta}\left(\sin \phi \operatorname{Im} h_{L}^{+}-\cos \phi \operatorname{Re} h_{L}^{+}\right)\right. \\
& \left.\left.+\frac{1}{\delta}\left(\cos \theta \operatorname{Re} h_{L}^{+}-\sin \theta \operatorname{Im} h_{L}^{+}\right)\right]\right\},
\end{aligned}
$$

where

$$
\begin{aligned}
\omega_{L, R} & =\sqrt{|\mathbf{k}|^{2}+M_{\tilde{t}_{L, R}}^{2}}, \\
\Delta & =\sqrt{\left(\Gamma_{L}+\Gamma_{R}\right)^{2}+\left(\omega_{L}-\omega_{R}\right)^{2}}, \\
\delta & =\sqrt{\left(\Gamma_{L}+\Gamma_{R}\right)^{2}+\left(\omega_{L}+\omega_{R}\right)^{2}}, \\
\tan \theta & =\frac{\omega_{L}+\omega_{R}}{\Gamma_{L}+\Gamma_{R}}, \quad \tan \phi=\frac{\omega_{L}-\omega_{R}}{\Gamma_{L}+\Gamma_{R}}, \\
h_{L, R}^{ \pm} & =\frac{\exp \left[\left(\omega_{L, R} \pm i \Gamma_{L, R}\right) / T\right]}{\left\{\exp \left[\left(\omega_{L, R} \pm i \Gamma_{L, R}\right) / T\right]-1\right\}^{2}}
\end{aligned}
$$

and where $\Gamma_{L, R}$ are the thermal widths for the $\tilde{t}_{L, R}$. The rates $\Gamma_{\tilde{t}}^{ \pm}$defined in Eq. (48) can then be expressed:

$$
\begin{aligned}
\Gamma_{\tilde{t}}^{+}= & -\frac{1}{T} \frac{y_{t}^{2}}{4 \pi^{2}}\left|A_{t} v_{u}(x)-\mu^{*} v_{d}(x)\right|^{2} \int_{0}^{\infty} \frac{k^{2} d k}{\omega_{R} \omega_{L}} \\
& \times\left\{\frac{1}{\Delta}\left[\sin \phi \operatorname{Im}\left(h_{L}^{+} \pm h_{R}^{+}\right)-\cos \phi \operatorname{Re}\left(h_{L}^{+} \mp h_{R}^{+}\right)\right]\right. \\
& \left.+\frac{1}{\delta}\left[\cos \theta \operatorname{Re}\left(h_{L}^{+} \mp h_{R}^{+}\right)-\sin \theta \operatorname{Im}\left(h_{L}^{+} \mp h_{R}^{+}\right)\right]\right\} .
\end{aligned}
$$

Meanwhile, the $C P$-violating source given in Eq. (48) can be expanded:

$$
\begin{aligned}
S_{\tilde{t}_{R}}^{C P}(x)= & N_{C} y_{t}^{2} \operatorname{Im}\left(\mu A_{t}\right) v(x)^{2} \dot{\beta}(x) \int_{0}^{\infty} \frac{k^{2} d k}{2 \pi^{2}} \frac{1}{\omega_{L} \omega_{R}} \\
& \times\left\{\frac { 1 } { \delta ^ { 2 } } \left[\operatorname{Re}\left(1+n_{R}^{+}+n_{L}^{+}\right) \sin 2 \theta\right.\right. \\
& \left.+\operatorname{Im}\left(n_{R}^{+}+n_{L}^{+}\right) \cos 2 \theta\right] \\
& +\frac{1}{\Delta^{2}}\left[\operatorname{Re}\left(n_{R}^{+}-n_{L}^{+}\right) \sin 2 \phi\right. \\
& \left.\left.-\operatorname{Im}\left(n_{R}^{+}+n_{L}^{+}\right) \cos 2 \phi\right]\right\},
\end{aligned}
$$

where $n_{L, R}^{ \pm}=n_{B}\left(\omega_{\tilde{t}_{L, R}} \pm \Gamma_{L, R}\right)$. Our result agrees with that of Ref. [8] except for a different relative sign in front of the $\cos 2 \phi$ term and the overall factor of $N_{C}$.

\section{Massive Fermions}

The $C P$-conserving rates for Higgsino-gaugino interactions given in Eq. (60) can be expanded:

$$
\begin{aligned}
\Gamma_{\tilde{H}^{ \pm}}^{ \pm}= & g_{2}^{2} v(x)^{2} \frac{1}{T} \int_{0}^{\infty} \frac{k^{2} d k}{2 \pi^{2}}\left(\frac{1}{\omega_{\tilde{H}} \omega_{\tilde{W}}}\right)\left(\frac { 1 } { \Delta } \left\{[ \omega _ { \tilde { H } } \omega _ { \tilde { W } } + \Gamma _ { \tilde { H } } \Gamma _ { \tilde { W } } - k ^ { 2 } + M _ { 2 } | \mu | \operatorname { c o s } \phi _ { \mu } \operatorname { s i n } 2 \beta ( x ) ] \left[\cos \phi \operatorname{Re}\left(h_{\tilde{W}}^{+} \mp h_{\tilde{H}}^{+}\right)\right.\right.\right. \\
& \left.\left.-\sin \phi \operatorname{Im}\left(h_{\tilde{W}}^{+} \pm h_{\tilde{H}}^{+}\right)\right]+\left[\Gamma_{\tilde{H}} \omega_{\tilde{W}}-\Gamma_{\tilde{W}} \omega_{\tilde{H}}\right]\left[\sin \phi \operatorname{Re}\left(h_{\tilde{W}}^{+} \mp h_{\tilde{H}}^{+}\right)+\cos \phi \operatorname{Im}\left(h_{\tilde{W}}^{+} \pm h_{\tilde{H}}^{+}\right)\right]\right\} \\
& +\frac{1}{\delta}\left\{\left[\omega_{\tilde{H}} \omega_{\tilde{W}}-\Gamma_{\tilde{H}} \Gamma_{\tilde{W}}+k^{2}-M_{2}|\mu| \cos \phi_{\mu} \sin 2 \beta(x)\right]\left[\cos \theta \operatorname{Re}\left(h_{\tilde{W}}^{+} \mp h_{\tilde{H}}^{+}\right)-\sin \theta \operatorname{Im}\left(h_{\tilde{W}}^{+} \mp h_{\tilde{H}}^{+}\right)\right]\right. \\
& \left.\left.-\left[\Gamma_{\tilde{H}} \omega_{\tilde{W}}+\Gamma_{\tilde{W}} \omega_{\tilde{H}}\right]\left[\cos \theta \operatorname{Im}\left(h_{\tilde{W}}^{+} \mp h_{\tilde{H}}^{+}\right)+\sin \theta \operatorname{Re}\left(h_{\tilde{W}}^{+} \mp h_{\tilde{H}}^{+}\right)\right]\right\}\right)
\end{aligned}
$$

where

$$
\begin{aligned}
\omega_{\tilde{H}, \tilde{W}} & =\sqrt{\mathbf{k}^{2}+M_{\tilde{H}, \tilde{W}}^{2}}, & \Delta & =\sqrt{\left(\Gamma_{\tilde{W}}+\Gamma_{\tilde{H}}\right)^{2}+\left(\omega_{\tilde{W}}-\omega_{\tilde{H}}\right)^{2}}, \quad \delta=\sqrt{\left(\Gamma_{\tilde{W}}+\Gamma_{\tilde{H}}\right)^{2}+\left(\omega_{\tilde{W}}+\omega_{\tilde{H}}\right)^{2}}, \\
\tan \theta & =\frac{\omega_{\tilde{W}}+\omega_{\tilde{H}}}{\Gamma_{\tilde{W}}+\Gamma_{\tilde{H}}}, & \tan \phi & =\frac{\omega_{\tilde{W}}-\omega_{\tilde{H}}}{\Gamma_{\tilde{W}}+\Gamma_{\tilde{H}}}, \quad h_{\tilde{W}, \tilde{H}}^{ \pm}=\frac{\exp \left[\left(\omega_{\tilde{W}, \tilde{H}} \pm i \Gamma_{\tilde{W}, \tilde{H}}\right) / T\right]}{\left\{\exp \left[\left(\omega_{\tilde{W}, \tilde{H}} \pm i \Gamma_{\tilde{W}, \tilde{H}}\right) / T\right]+1\right\}^{2}} .
\end{aligned}
$$

The $C P$-violating Higgsino source in Eq. (64) can be expressed: 


$$
\begin{aligned}
S_{\tilde{H}^{ \pm}}^{Q P}(x)= & 2 g_{2}^{2} M_{2} \operatorname{Im}(\mu) v(x)^{2} \dot{\beta} \int_{0}^{\infty} \frac{k^{2} d k}{2 \pi^{2}}\left(\frac{1}{\omega_{\tilde{H}} \omega_{\tilde{W}}}\right)\left\{\frac{1}{\Delta^{2}}\left[\sin 2 \phi \operatorname{Re}\left(N_{\tilde{W}}^{+}-N_{\tilde{H}}^{+}\right)+\cos 2 \phi \operatorname{Im}\left(N_{\tilde{W}}^{+}+N_{\tilde{H}}^{+}\right)\right]\right. \\
& \left.+\frac{1}{\delta^{2}}\left[\sin 2 \theta \operatorname{Re}\left(1-N_{\tilde{W}}^{+}-N_{\tilde{H}}^{+}\right)-\cos 2 \theta \operatorname{Im}\left(N_{\tilde{W}}^{+}+N_{\tilde{H}}^{+}\right)\right]\right\},
\end{aligned}
$$

where $N_{\tilde{H}, \tilde{W}}^{+}=n_{B}\left(\omega_{\tilde{H}, \tilde{W}} \pm i \Gamma_{\tilde{H}, \tilde{W}}\right)$. Our result agrees with that of Ref. [8] except for the sign of the $\cos 2 \phi$ term.

\section{Chiral Fermions}

For chiral fermions, the $C P$-conserving chirality-changing rates in Eq. (66) can be expanded:

$$
\begin{aligned}
\Gamma_{t_{R}}^{ \pm}= & \frac{1}{T} \frac{N_{C} y_{t} v_{u}^{2}}{\pi^{2}} \int_{0}^{\infty} k^{2} d k\left\{\frac { Z _ { p } ^ { R } Z _ { p } ^ { L } } { \delta _ { p } } \left[\sin \theta_{p}\left\{\operatorname{Re}\left(\lambda_{p}^{L} h_{p L}^{+} \mp \lambda_{p}^{R} h_{p R}^{+}\right)-\operatorname{Im}\left(h_{p L}^{+} \mp h_{p R}^{+}\right)\right\}+\cos \theta_{p} \operatorname{Re}\left(h_{p L}^{+} \mp h_{p R}^{+}\right)\right.\right. \\
& \left.+\frac{T}{\delta_{p}} \cos 2 \theta_{p}\left(\lambda_{p}^{L} \mp \lambda_{p}^{R}\right) \operatorname{Re}\left(1-N_{p L}^{+}-N_{p R}^{+}\right)\right]-\frac{Z_{p}^{L} Z_{h}^{R}}{\Delta_{h p}}\left[\sin \phi_{h p}\left\{\operatorname{Re}\left(\lambda_{p}^{L} h_{p L}^{+} \pm \lambda_{h}^{R} h_{h R}^{+}\right)-\operatorname{Im}\left(h_{p L}^{+} \pm h_{h R}^{+}\right)\right\}\right. \\
& \left.\left.-\cos \phi_{h p} \operatorname{Re}\left(h_{p L}^{+} \mp h_{h R}^{+}\right)+\frac{T}{\Delta_{h p}} \cos 2 \phi_{h p}\left(\lambda_{p}^{L} \pm \lambda_{h}^{R}\right) \operatorname{Re}\left(N_{p L}^{+}-N_{h R}^{+}\right)\right]+(p \leftrightarrow h)\right\}
\end{aligned}
$$

where

$$
\begin{aligned}
\delta_{p} & =\sqrt{\left(\omega_{p}^{R}+\omega_{p}^{L}\right)^{2}+\left(\Gamma_{p}^{R}+\Gamma_{p}^{L}\right)^{2}}, \\
\Delta_{h p} & =\sqrt{\left(\omega_{p}^{L}-\omega_{h}^{R}\right)^{2}+\left(\Gamma_{h}^{R}+\Gamma_{p}^{L}\right)^{2}}, \\
h_{p L}^{ \pm} & =h_{F}\left(\omega_{p}^{L} \pm i \Gamma_{p}^{L}\right), \text { etc., } \\
N_{p L}^{ \pm} & =n_{F}\left(\omega_{p}^{L} \pm i \Gamma_{p}^{L}\right), \text { etc., } \quad \tan \theta_{p}=\frac{\omega_{p}^{L}+\omega_{p}^{R}}{\Gamma_{p}^{L}+\Gamma_{p}^{R}} \\
\tan \phi_{h p} & =\frac{\omega_{h}^{R}-\omega_{p}^{L}}{\Gamma_{p}^{L}+\Gamma_{h}^{R}},
\end{aligned}
$$

and where the

$$
\lambda_{p, h}^{L, R}=\frac{\partial \Gamma_{p, h}^{L, R}}{\partial \mu_{t_{L, R}}},
$$

parametrize the linear shifts in the thermal widths due to nonvanishing chemical potential. As noted at the end of Appendix A, in a fully resummed calculation of the fermion self-energy, such shifts which are linear in $\mu_{i} / T$ may arise, and thus have to be included in our calculations, which we defer to future work. Also note that we have approximated the residues $Z_{p, h}^{L, R}$ to be purely real, which is true at the order we are working.
[1] A. D. Sakharov, Pis'ma Zh. Eksp. Teor. Fiz. 5, 32 (1967) [JETP Lett. 5, 24 (1967)].

[2] Particle Data Group Collaboration, S. Eidelman et al., Phys. Lett. B 592, 1 (2004).

[3] WMAP Collaboration, D. N. Spergel et al., Astrophys. J. Suppl. Ser. 148, 175 (2003).

[4] M. Dine and A. Kusenko, Rev. Mod. Phys. 76, 1 (2004).

[5] J. Kang, P. Langacker, T. J. Li, and T. Liu, Phys. Rev. Lett. 94, 061801 (2005).

[6] J. M. Cline, K. Kainulainen, and K. A. Olive, Phys. Rev. D 49, 6394 (1994).

[7] A. G. Cohen, D. B. Kaplan, and A. E. Nelson, Phys. Lett. B 336, 41 (1994).

[8] A. Riotto, Phys. Rev. D 58, 095009 (1998).

[9] J. Schwinger, J. Math. Phys. (N.Y.) 2, 407 (1961); K. T. Mahanthappa, Phys. Rev. 126, 329 (1962); P. M. Bakshi and K. T. Mahanthappa, J. Math. Phys. (N.Y.) 4, 1 (1963); 4, 12 (1963); L. V. Keldysh, Zh.
Eksp. Teor. Fiz. 47, 1515 (1964) [Sov. Phys. JETP 20, 1018 (1965)]; R. A. Craig, J. Math. Phys. (N.Y.) 9, 605 (1968); K. c. Chou, Z. b. Su, B.1. Hao, and L. Yu, Phys. Rep. 118, 1 (1985).

[10] P. Huet and A. E. Nelson, Phys. Rev. D 53, 4578 (1996).

[11] M. Carena, J.M. Moreno, M. Quiros, M. Seco, and C. E. M. Wagner, Nucl. Phys. B 599, 158 (2001).

[12] M. Carena, M. Quiros, M. Seco, and C.E. M. Wagner, Nucl. Phys. B 650, 24 (2003).

[13] M. Joyce, T. Prokopec, and N. Turok, Phys. Rev. D 53, 2930 (1996).

[14] M. Le Bellac, Thermal Field Theory (Cambridge University Press, Cambridge, England, 1996).

[15] E. J. Levinson and D. H. Boal, Phys. Rev. D 31, 3280 (1985); J. P. Blaizot and J. Y. Ollitrault, Phys. Rev. D 48, 1390 (1993); O. K. Kalashnikov, Mod. Phys. Lett. A 12, 347 (1997); JETP Lett. 67, 1 (1998); Phys. Scr. 58, 310 (1998); Mod. Phys. Lett. A 13, 1719 (1998); Phys. Scr. 60, 131 (1999). 
[16] H. A. Weldon, Phys. Rev. D 40, 2410 (1989).

[17] V. V. Klimov, Yad. Fiz. 33, 1734 (1981) [Sov. J. Nucl. Phys. 33, 934 (1981)]; Zh. Eksp. Teor. Fiz. 82, 336 (1982) [Sov. Phys. JETP 55, 199 (1982)].

[18] H. A. Weldon, Phys. Rev. D 61, 036003 (2000).

[19] E. Braaten and R. D. Pisarski, Nucl. Phys. B 337, 569 (1990).

[20] E. Braaten and R. D. Pisarski, Phys. Rev. D 45, R1827 (1992).

[21] E. Braaten and R. D. Pisarski, Phys. Rev. D 46, 1829 (1992).

[22] K. Enqvist, A. Riotto, and I. Vilja, Phys. Lett. B 438, 273 (1998).

[23] P. Elmfors, K. Enqvist, A. Riotto, and I. Vilja, Phys. Lett. B 452, 279 (1999).

[24] S. P. Martin, hep-ph/9709356.

[25] T. Prokopec, M. G. Schmidt, and S. Weinstock, Ann. Phys. (N.Y.) 314, 208 (2004); Ann. Phys. (N.Y.) 314, 267 (2004).

[26] M. Carena, M. Quiros, A. Riotto, I. Vilja, and C.E. M. Wagner, Nucl. Phys. B 503, 387 (1997).

[27] G. F. Giudice and M. E. Shaposhnikov, Phys. Lett. B 326, 118 (1994).

[28] G. D. Moore, Phys. Lett. B 412, 359 (1997).

[29] J. M. Cline, M. Joyce, and K. Kainulainen, J. High Energy Phys. 07 (2000) 018.
[30] D. Bodeker, G. D. Moore, and K. Rummukainen, Phys. Rev. D 61, 056003 (2000); G.D. Moore and K. Rummukainen, Phys. Rev. D 61, 105008 (2000); G. D. Moore, Phys. Rev. D 62, 085011 (2000).

[31] M. Carena, M. Quiros, and C. E. M. Wagner, Nucl. Phys. B 524, 3 (1998).

[32] M. Laine and K. Rummukainen, Nucl. Phys. B 535, 423 (1998).

[33] J. M. Moreno, M. Quiros, and M. Seco, Nucl. Phys. B 526, 489 (1998).

[34] B.C. Regan, E.D. Commins, C. J. Schmidt, and D. DeMille, Phys. Rev. Lett. 88, 071805 (2002).

[35] M. V. Romalis, W.C. Griffith, J.P. Jacobs, and E. N. Fortson, Phys. Rev. Lett. 86, 2505 (2001).

[36] T. Falk, K. A. Olive, M. Pospelov, and R. Roiban, Nucl. Phys. B 560, 3 (1999).

[37] T. Ibrahim and P. Nath, Phys. Rev. D 57, 478 (1998) 58, 019901(E) (1998); 60, 079903(E); 60, 119901(E).

[38] J. Hisano and Y. Shimizu, Phys. Rev. D 70, 093001 (2004).

[39] J. Erler and M. J. Ramsey-Musolf, Prog. Part. Nucl. Phys. 54, 351 (2005).

[40] C. Balazs, M. Carena, A. Menon, D. E. Morrissey, and C. E. M. Wagner, Phys. Rev. D 71, 075002 (2005). 